\title{
RAPOPORT-ZINK UNIFORMIZATION OF HODGE-TYPE SHIMURA VARIETIES
}

\author{
WANSU KIM \\ Department of Mathematics, KIAS, 85 Hoegiro, Dongdaemun-gu, Seoul 02455, South Korea; \\ email: wansukim@kias.re.kr
}

Received 31 December 2015; accepted 2 August 2018

\begin{abstract}
We show that the integral canonical models of Hodge-type Shimura varieties at odd good reduction primes admits ' $p$-adic uniformization' by Rapoport-Zink spaces of Hodge type constructed in Kim [Forum Math. Sigma 6 (2018) e8, 110 MR 3812116].

2010 Mathematics Subject Classification: 11G18 (primary); 14G35 (secondary)
\end{abstract}

\section{Contents}

1 Introduction

2 Notation and preliminaries $\quad 4$

3 Review on Shimura varieties of Hodge type 11

4 Rapoport-Zink uniformization via formal schemes 21

5 Rapoport-Zink uniformization via rigid geometry 32

References

(c) The Author 2018. This is an Open Access article, distributed under the terms of the Creative Commons Attribution licence (http://creativecommons.org/licenses/by/4.0/), which permits unrestricted re-use, distribution, and reproduction in any medium, provided the original work is properly cited. 


\section{Introduction}

Shimura varieties have many interesting structures and symmetries which encode arithmetic information. It is now a standard folklore conjecture that the cohomology of Shimura varieties should realize the global Langlands correspondence. It is natural to look for a purely local analogue of Shimura varieties, whose cohomology should realize the local Langlands correspondence, and ask how the local-global compatibility is encoded geometrically. For example, Carayol [4] showed that the (height-2) Lubin-Tate tower plays the role of 'local Shimura varieties' and the identification of the Lubin-Tate tower with the completion of the modular tower at a supersingular point (by Serre-Tate deformation theory) encodes the local-global compatibility.

Many interesting examples of Shimura varieties can be understood as moduli spaces of certain polarised abelian varieties equipped with the action of some semisimple algebra and level structure. Such Shimura varieties are called of PEL type, and examples include modular curves, Siegel modular varieties, and unitary Shimura varieties. The purely local analogue of PEL Shimura varieties was constructed by Rapoport and Zink [26], which are now called Rapoport-Zink spaces of EL or PEL type. In the good reduction case, Rapoport-Zink spaces are moduli spaces of $p$-divisible groups with some action of semisimple algebra (and possibly with polarization), up to rigidification (by quasi-isogeny). Furthermore, they showed the relationship between certain Rapoport-Zink spaces of (P)EL type and PEL Shimura varieties in a way that is analogous to the complex analytic uniformization of Shimura varieties and generalizes some known examples (of modular and Shimura curves via Lubin-Tate and Drinfeld towers); cf. [26, Ch. 6]. We call this result the Rapoport-Zink uniformization of PEL Shimura varieties.

There is a more general class of Shimura varieties which, over $\mathbb{C}$, parametrize abelian varieties with certain Hodge cycles. They are called Shimura varieties of Hodge type. An example naturally comes up in relation to the construction of an abelian variety associated to a polarized complex K3 surfaces (due to Kuga and Satake). Although such moduli spaces are essentially defined only in characteristic 0 (as Hodge cycles are defined using singular cohomology with $\mathbb{Q}$-coefficients), recent developments in integral $p$-adic Hodge theory allow us to study certain 'natural' integral models of such Shimura varieties at odd good reduction primes. See Kisin [14] and Vasiu [27-29] for the statement and the proof.

In the author's previous work [12], the local analogue of Hodge-type Shimura varieties for $p>2$ (called Rapoport-Zink spaces of Hodge type) was constructed under a certain unramifiedness assumption, generalizing the construction of Rapoport-Zink spaces of (P)EL type; loosely speaking, Rapoport-Zink spaces 
of Hodge type can be thought of as moduli spaces of $p$-divisible groups with Tate tensors (instead of endomorphisms and polarization) up to rigidification by quasiisogeny. In this paper, we prove the Hodge-type generalization of the RapoportZink uniformization for odd good reduction prime.

Let $(G, \mathfrak{H})$ be a Shimura datum of Hodge type (with $G$ connected), and assume that $G$ admits a reductive $\mathbb{Z}_{(p)}$-model for $p>2$, also denoted as $G$. Let $E:=E(G, \mathfrak{H})$ denote the reflex field, and we choose a prime $\mathfrak{p}$ over $p$, which is necessarily unramified. Then the aforementioned result of Vasiu and Kisin produces an 'integral canonical model' $\mathscr{S}_{\mathrm{K}}$ of $\mathrm{Sh}_{\mathrm{K}}(G, \mathfrak{H})$, where $\mathrm{K}=\mathrm{K}_{p} \mathrm{~K}^{p}$ with $\mathrm{K}_{p}=G\left(\mathbb{Z}_{p}\right)$ and $\mathrm{K}^{p} \subset G\left(\mathbb{A}_{\mathrm{f}}^{p}\right)$ is a 'small enough' open compact subgroup.

Let $W:=W\left(\overline{\mathbb{F}}_{p}\right)$ and $K_{0}:=$ Frac $W$, viewed as a $\mathscr{O}_{E, \mathfrak{p}}$-algebra. We choose $x \in \mathscr{S}_{\mathrm{K}}\left(\overline{\mathbb{F}}_{p}\right)$. From $x$ we can (noncanonically) choose an element $b \in G\left(K_{0}\right)$ from the Frobenius operator on the Dieudonné module of the abelian variety with extra structure corresponding to $x$, and let $\mathrm{RZ}_{G, b}$ denote the Rapoport-Zink space of Hodge type [12] associated to $(G, b)$; see Section 4.1 for the details.

Theorem 1.1 (cf. Theorems 4.7, 5.4). There exists an isomorphism of formal schemes over $W$

$$
\Theta^{\phi}: I^{\phi}(\mathbb{Q}) \backslash \mathrm{RZ} Z_{G, b} \times G\left(\mathbb{A}_{\mathrm{f}}^{p}\right) / \mathrm{K}^{p} \rightarrow\left(\mathscr{S}_{\mathrm{K}, W}\right)_{/ \mathscr{I}^{\phi},}
$$

where $I^{\phi}(\mathbb{Q})$ is the group of self quasi-isogenies of abelian varieties with tensors coming from a closed point of $\mathscr{S}_{\mathrm{K}}$. The target of the isomorphism is the completion of $\mathscr{S}_{\mathrm{K}, W}$ at the isogeny leaf $\mathscr{I}^{\phi}$ defined in Example 4.6.1. Furthermore, the isomorphism $\Theta^{\phi}$ naturally descends over $\mathscr{O}_{E, \mathfrak{p}}$, and on the rigid analytic generic fibres the isomorphism extends to a $G\left(\mathbb{A}_{\mathrm{f}}\right)$-equivariant isomorphism of towers on the both sides.

When $b$ is basic, we expect a stronger version of the theorem to hold; namely, if $b$ is basic then the isogeny leaf equals the basic Newton stratum and $I^{\phi}$ is an inner form of $G$. This is proved in [32, Corollary 7.2.16]. (The proof that appeared in the earlier version of this paper had a gap. The author appreciates Xinwen Zhu for informing me of this.)

Let us make a remark on the proof. Unlike the PEL case, $\mathscr{S}_{\mathrm{K}}$ does not have a good moduli interpretation and this causes number of additional difficulties.

First, it is not trivial to construct the morphism $\mathrm{RZ}_{G, b} \rightarrow \widehat{\mathscr{S}}_{\mathrm{K}, W}$, where the target is the $p$-adic completion of $\mathscr{S}_{\mathrm{K}, W}$. To overcome this problem, we use a deformation-theoretic trick, exploiting that the completions of $\widehat{\mathscr{S}}_{\mathrm{K}, W}$ at closed points are well-understood by construction, and the work of Chen, Kisin and Viehmann [5] which allows us to control the connected components of $\mathrm{RZ}_{G, b}$. See Proposition 4.3 and subsequent remarks for more details. Now one can repeat the proof of Theorems 6.21 and 6.23 in [26] to obtain this theorem. 
Recently, Howard and Pappas [9] gave another construction (using global techniques) of Hodge-type Rapoport-Zink spaces that come from global Hodgetype Shimura data, in such a way that the Rapoport-Zink uniformization holds by construction. Indeed, their construction relies on the existence of integral canonical models of Hodge-type Shimura varieties and the Rapoport-Zink uniformization for Siegel modular varieties, and the Hodge-type Rapoport-Zink uniformization is obtained by pulling back the Siegel case of Rapoport-Zink uniformization. Note that some of the ideas in this paper are used in [9] for the construction of Hodge-type Rapoport-Zink spaces. Our approach is to construct Hodge-type Rapoport-Zink spaces by purely local means in [12], and separately obtain the link with the global theory (that is, Rapoport-Zink uniformization).

We have excluded the case of $p=2$ because the purely local construction of Rapoport-Zink spaces of Hodge type given in [12] requires $p>2$. (Note that the 2-adic integral canonical models were constructed in [13].) It is also natural to expect that the purely local construction of Rapoport-Zink spaces of Hodge type as well as the Rapoport-Zink uniformization can be extended to the case when $p=2$ (which is the author's work in progress).

The Rapoport-Zink uniformization is more interesting in the bad reduction case, but we do not consider this case as the construction of Rapoport-Zink spaces in [12] has not been generalized in the bad reduction case. On the other hand, the recent work of Kisin and Pappas on integral models of Hodge-type Shimura varieties with parahoric level structure [16] suggests that the uniformization result can be generalized to some bad reduction cases.

In Sections 2 and 3, we review basic notions and set up the notationSection 2 is for general notions, and Section 3 is for Shimura varieties and Rapoport-Zink spaces of Hodge type. In Section 4, we obtain the Rapoport-Zink uniformization at the hyperspecial maximal level at $p$, and in Section 5, we extend the uniformization to rigid analytic towers.

\section{Notation and preliminaries}

2.1. For any ring $R$, an $R$-module $M$, and an $R$-algebra $R^{\prime}$, we write $M_{R^{\prime}}:=$ $R^{\prime} \otimes_{R} M$. Similarly, if $R$ is a noetherian adic ring and $\mathfrak{X}$ is a formal scheme over Spf $R$, then for any continuous morphism of adic rings $R \rightarrow R^{\prime}$ we write $\mathfrak{X}_{R^{\prime}}:=$ $\mathfrak{X} \times$ Spf $R$ Spf $R^{\prime}$.

2.2. For definitions in category theory, see [31] and references therein. Let $\mathcal{C}$ be a pseudo-abelian symmetric tensor category such that arbitrary (infinite) direct sum exists. Let 1 denote the identity object for $\otimes$-product in $\mathcal{C}$, which exists by the axioms of tensor categories. (Pseudo-abelian categories are defined in the same 
way as abelian categories, except that we only require the existence of kernel for idempotent morphisms instead of requiring the existence of kernel and cokernel for any morphism. In practice, the pseudo-abelian categories that we encounter are the category of filtered or graded objects in some abelian category.)

Let $\mathcal{D}$ be a full subcategory of $\mathcal{C}$ which is stable under direct sums, tensor products, and direct factors. Assume furthermore that $\mathcal{D}$ is rigid; that is, every object of $\mathcal{D}$ has a dual. (For example, $\mathcal{C}$ can be the category of $R$-modules filtered by direct factors, and $\mathcal{D}$ can be the full subcategory of finitely generated projective $R$-modules.) Then for any object $M \in \mathcal{D}$, we let

$$
M^{\otimes} \in \mathcal{C}
$$

denote the direct sum of any (finite) combination of tensor products, symmetric products, alternating products, and duals of $M$. Note that we naturally have

$$
M^{\otimes}=\left(M^{*}\right)^{\otimes} .
$$

2.3. Let $S$ be a (not necessarily connected) scheme, and $\bar{x}$ a geometric point of $S$. Then $\pi_{1}^{\text {et }}(S, \bar{x})$ denotes the étale fundamental group of the connected component of $S$ containing $\bar{x}$.

2.4. Abelian Schemes. For any abelian scheme $f: \mathcal{A} \rightarrow S$ (where $S$ is any scheme), we define

$$
\begin{aligned}
& \mathcal{V}_{\mathrm{dR}}(\mathcal{A})\left(=\mathcal{V}_{\mathrm{dR}}(\mathcal{A} / S)\right):=\mathrm{H}_{\mathrm{dR}}^{1}(\mathcal{A} / S)^{*} \\
& \mathcal{V}_{\mathbb{A}_{\mathrm{f}}}(\mathcal{A})\left(=\mathcal{V}_{\mathbb{A}_{\mathrm{f}}}(\mathcal{A} / S)\right):=\prod_{\ell \neq \infty}^{\prime}\left(R^{1} f_{\mathrm{ét} *} \mathbb{Q}_{\ell}\right)^{*}
\end{aligned}
$$

where $\prod^{\prime}$ is the restricted product with respect to $\left\{\left(R^{1} f_{\text {ét } *} \mathbb{Z}_{\ell}\right)^{*}\right\}$. Note that (if $S$ is connected then) for any geometric point $\bar{x}$ of $S$ the fibre $\mathcal{V}_{\mathbb{A}_{\mathrm{f}}}(\mathcal{A})_{\bar{x}}$ has a natural continuous action of $\pi_{1}^{\text {et }}(S, \bar{x})$.

For any prime $p$, we can define the $p$-component $\mathcal{V}_{\mathbb{Q}_{p}}(\mathcal{A}):=\left(R^{1} f_{\text {ét } * \mathbb{Q}_{p}}\right)^{*}$ and the prime-to- $p$ component $\mathcal{V}_{\mathbb{A}_{\mathrm{f}}^{p}}(\mathcal{A})$ with

$$
\mathcal{V}_{\mathbb{A}_{\mathrm{f}}}(\mathcal{A})=\mathcal{V}_{\mathbb{A}_{\mathrm{f}}^{p}}(\mathcal{A}) \times \mathcal{V}_{\mathbb{Q}_{p}}(\mathcal{A})
$$

With suitably chosen $\mathcal{C}$ and $\mathcal{D}$, we can form $\mathcal{V}_{\mathrm{dR}}(\mathcal{A})^{\otimes}, \mathcal{V}_{\mathrm{A}_{\mathrm{f}}}(\mathcal{A})^{\otimes}$, and so on, as in Section 2.2.

DEFINITION 2.4.2. Let $\mathcal{A}$ be an abelian scheme over some scheme $S$. A de Rham tensor on $\mathcal{A}$ is a filtered $\mathcal{O}_{S}$-morphism $t_{\mathrm{dR}}: \mathbf{1} \rightarrow \mathcal{V}_{\mathrm{dR}}(\mathcal{A})^{\otimes}$, where $\mathbf{1}$ is $\mathcal{O}_{S}$ equipped 
with the filtration whose grading is concentrated in 0 . We often abuse the notation and denote by $t_{\mathrm{dR}} \in \Gamma\left(S, \mathcal{V}_{\mathrm{dR}}(\mathcal{A})^{\otimes}\right)$ the image of $1 \in \Gamma\left(S, \mathcal{O}_{S}\right)$ by $t_{\mathrm{dR}}: \mathbf{1} \rightarrow$ $\mathcal{V}_{\mathrm{dR}}(\mathcal{A})$

An étale tensor on $\mathcal{A}$ is an $\mathbb{A}_{\mathrm{f}}$-linear morphism $t_{\text {ét }}: \mathbf{1} \rightarrow \mathcal{V}_{\mathbb{A}_{\mathrm{f}}}(\mathcal{A})^{\otimes}$ of étale sheaves on $S$, where 1 is the constant $\mathbb{A}_{\mathrm{f}}$-local system of rank 1 . We similarly define a prime-to-p étale tensor $t_{\mathrm{et}}^{p}: \mathbf{1} \rightarrow \mathcal{V}_{\mathbb{A}_{\mathrm{f}}^{p}}(\mathcal{A})^{\otimes}$ and a $p$-adic étale tensor $t_{\text {ét, } p}: \mathbf{1} \rightarrow \mathcal{V}_{\mathbb{Q}_{p}}(\mathcal{A})^{\otimes}$.

If $S$ is a smooth variety over $\mathbb{C}$, then we can also define the following $\mathbb{Q}$-local system

$$
\mathcal{V}(\mathcal{A})(=\mathcal{V}(\mathcal{A} / S)):=\left(R^{1} f_{*}^{\mathrm{an}} \mathbb{Q}\right)^{*}
$$

and we have natural isomorphisms $\mathbb{A}_{\mathrm{f}} \otimes_{\mathbb{Q}} \mathcal{V}(\mathcal{A}) \cong \mathcal{V}_{\mathbb{A}_{\mathrm{f}}}(\mathcal{A})$ and $\mathcal{O}_{S} \otimes_{\mathbb{Q}} \mathcal{V}(\mathcal{A}) \cong$ $\mathcal{V}_{\mathrm{dR}}(\mathcal{A})$. By classical Hodge theory, we obtain a variation of $\mathbb{Q}$-Hodge structures.

With suitably chosen $\mathcal{C}$ and $\mathcal{D}$, we can form $\mathcal{V}(\mathcal{A})^{\otimes}$ as in Section 2.2. Given a $\mathbb{Q}$-linear morphism of locally constant sheaves $t_{B}: \mathbf{1} \rightarrow \mathcal{V}(\mathcal{A})^{\otimes}$ (where $\mathbf{1}$ is the constant sheaf $\mathbb{Q}$ on $S$ ), we define the étale and de Rham components $t_{\mathrm{e} t}$ and $t_{\mathrm{dR}}$ of $t_{B}$ as follows:

$$
\begin{gathered}
t_{\text {ét }}: \mathbf{1} \stackrel{\stackrel{\mathbb{A}_{\mathrm{f}} \otimes t_{B}}{\longrightarrow}}{\longrightarrow} \mathbb{A}_{\mathrm{f}} \otimes_{\mathbb{Q}} \mathcal{V}(\mathcal{A})^{\otimes} \stackrel{\sim}{\rightarrow} \mathcal{V}_{\mathbb{A}_{\mathrm{f}}}(\mathcal{A})^{\otimes} \\
t_{\mathrm{dR}}: \mathbf{1} \stackrel{\mathcal{O}_{S} \otimes t_{B}}{\longrightarrow} \mathcal{O}_{S} \otimes_{\mathbb{Q}} \mathcal{V}(\mathcal{A})^{\otimes} \stackrel{\sim}{\rightarrow} \mathcal{V}_{\mathrm{dR}}(\mathcal{A})^{\otimes} .
\end{gathered}
$$

Clearly $t_{\text {ét }}$ is an étale tensor, and we are often interested in $t_{B}$ such that $t_{\mathrm{dR}}$ lies in the 0th filtration for the Hodge filtration. Such $t_{B}$ can be thought of as a 'family of Hodge tensors'.

2.5. Group theory preliminaries. Throughout this section, let $R$ be either a field of characteristic zero or a discrete valuation ring of mixed characteristic. In practice, $R$ will be one of $\mathbb{Q}, \mathbb{Z}_{(p)}$, and $\mathbb{Z}_{p}$. Let $G$ be a reductive group over $R$; that is, an affine smooth group scheme over $R$ such that all the fibres are reductive groups. Let $M$ be a free $R$-module of finite rank, and we fix a closed immersion of group schemes $G \hookrightarrow \mathrm{GL}_{R}(M)$. Let $M^{\otimes}$ be as defined in Section 2.2, where $\mathcal{C}$ is the category of $R$-modules and $\mathcal{D}$ is the category of locally free $R$-modules of finite rank.

Proposition 2.5.1. In the above setting, here exists a finitely many elements $s_{\alpha} \in$ $M^{\otimes}$ such that $G$ is the pointwise stabilizer of $\left(s_{\alpha}\right)$; that is, for any $R$-algebra $R^{\prime}$, we have

$$
G\left(R^{\prime}\right)=\left\{g \in \mathrm{GL}_{R}(M)\left(R^{\prime}\right) ; g\left(s_{\alpha}\right)=s_{\alpha} \forall \alpha\right\} .
$$

Proof. The case when $R$ is a field is proved in [7, Proposition 3.1], and the case of discrete valuation rings is proved in [14, Proposition 1.3.2]. 
EXAMPLE 2.5.2. If $G$ is a 'classical group' then one can often explicitly write down $\left(s_{\alpha}\right)$ that define $G$ in the sense of Proposition 2.5.1. For example, for a perfect alternating form $\psi: M \otimes M \rightarrow R$ on a projective $R$-module $M$, we can find a tensor $s_{\psi} \in M^{\otimes}$ whose pointwise stabilizer is $\operatorname{GSp}(M, \psi)$, which is explained in [12, Example 2.1.4].

DEFINITION 2.5.3. Let $\mathfrak{X}$ be an $R$-scheme (or a formal scheme over $R$ ). For a cocharacter $\mu: \mathbb{G}_{m} \rightarrow \mathrm{GL}_{R}(M)_{\mathfrak{X}}$, we say that a grading $\operatorname{gr} \bullet\left(\mathcal{O}_{\mathfrak{X}} \otimes_{R} M\right)$ is induced from $\mu$ if the $\mathbb{G}_{m}$-action on $\mathcal{O}_{\mathfrak{X}} \otimes_{R} M$ via $\mu$ leaves each grading stable, and the resulting $\mathbb{G}_{m}$-action on $\mathrm{gr}^{a}\left(\mathcal{O}_{\mathfrak{X}} \otimes_{R} M\right)$ is given by

$$
\mathbb{G}_{m} \stackrel{z \mapsto z^{-a}}{\longrightarrow} \mathbb{G}_{m} \stackrel{z \mapsto z \text { id }}{\longrightarrow} \operatorname{GL}\left(g r^{a}\left(\mathcal{O}_{\mathfrak{X}} \otimes_{R} M\right)\right) .
$$

We additionally fix finitely many $\left(s_{\alpha}\right) \subset M^{\otimes}$ defining $G \subset \mathrm{GL}_{R}(M)$. Let $\mathscr{E}$ be a vector bundle on $\mathfrak{X}$. Then we can form $\mathscr{E} \otimes$ in the category of quasicoherent sheaves. For (finitely many) global sections $\left(t_{\alpha}\right) \subset \Gamma(\mathfrak{X}, \mathscr{E} \otimes)$, we define the following scheme over $\mathfrak{X}$

$$
P_{\mathfrak{X}}:=\operatorname{isom}_{\mathcal{O}_{\mathfrak{X}}}\left(\left[\mathscr{E},\left(t_{\alpha}\right)\right],\left[\mathcal{O}_{\mathfrak{X}} \otimes_{R} M,\left(1 \otimes s_{\alpha}\right)\right]\right) \subset \operatorname{isom}_{\mathcal{O}_{\mathfrak{X}}}\left(\mathscr{E}, \mathcal{O}_{\mathfrak{X}} \otimes_{R} M\right),
$$

which classifies isomorphisms of vector bundles over $\mathfrak{X}$ which match $\left(t_{\alpha}\right)$ and

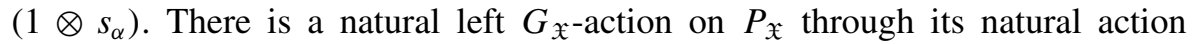
on $\mathcal{O}_{\mathfrak{X}} \otimes_{R} M$. Note that $P_{\mathfrak{X}}$ is a trivial $G$-torsor if and only if there exists an isomorphism $\varsigma: \mathscr{E} \stackrel{\sim}{\rightarrow} \mathcal{O}_{\mathfrak{X}} \otimes_{R} M$ which matches $\left(t_{\alpha}\right)$ and $\left(1 \otimes s_{\alpha}\right)$. Indeed, such $\varsigma$ defines a section $\mathfrak{X} \rightarrow P_{\mathfrak{X}}$ and any other sections are translates by the $G$-action.

From now on, assume that $R$ is either a field or a henselian discrete valuation ring, and let $R^{\mathrm{sh}}$ denote the strict henselization of $R$. (If $R$ is a field, then $R^{\mathrm{sh}}$ is its algebraic closure. If $R=W\left(\overline{\mathbb{F}}_{p}\right)$ then $R=R^{\text {sh }}$.) For a cocharacter $\mu: \mathbb{G}_{m} \rightarrow G_{R^{\text {sh }}}$ and $g \in G\left(R^{\text {sh }}\right)$, we write ${ }^{g} \mu:=g \mu g^{-1}$ and let $\{\mu\}:=\left\{{ }^{g} \mu: g \in G\left(R^{\text {sh }}\right)\right\}$ denote the $G\left(R^{\text {sh }}\right)$-conjugacy class of $\mu: \mathbb{G}_{m} \rightarrow G_{R^{\text {sh }}}$. We assume that the conjugacy class $\{\mu\}$ is defined over $R$.

The following terminology of ' $\{\mu\}$-filtrations' is a slight generalization of [12, Definition 2.2.3].

DEFINITION 2.5.5. Let $\mathfrak{X}$ be a scheme (or a formal scheme) over $R$. Let $\mathscr{E}$ be a vector bundle over $\mathfrak{X}$, with $\left(t_{\alpha}\right) \subset \Gamma(\mathfrak{X}, \mathscr{E} \otimes)$.

First, assume that $P_{\mathfrak{X}}$, defined in (2.5.4), is a trivial $G$-torsor and $\{\mu\}$ contains a cocharacter $\mu$ defined over $R$. Then a filtration $\mathrm{Fil}^{\bullet} \mathscr{E}$ of $\mathscr{E}$ is called a $\{\mu\}$-filtration (with respect to $\left(t_{\alpha}\right)$ ) if there exists an isomorphism $\varsigma: \mathscr{E} \stackrel{\sim}{\rightarrow}$ $\mathcal{O}_{\mathfrak{X}} \otimes_{R} M$, matching $\left(t_{\alpha}\right)$ and $\left(1 \otimes s_{\alpha}\right)$, that takes $\mathrm{Fil} \bullet \mathscr{E}$ to a filtration of $\mathcal{O}_{\mathfrak{X}} \otimes_{R} M$ induced by $\mu$ for some $\mu \in\{\mu\}$. 
When $P_{\mathfrak{X}}$ is a $G$-torsor, a filtration $\mathrm{Fil} \bullet \mathscr{E}$ of $\mathscr{E}$ is called a $\{\mu\}$-filtration (with respect to $\left.\left(t_{\alpha}\right)\right)$ if it is étale-locally a $\{\mu\}$-filtration; in other words, there exists an étale covering $f: \mathfrak{Y} \rightarrow \mathfrak{X}$ such that $P_{\mathfrak{Y}}$ is a trivial $G$-torsor and $\left(\mathrm{F} i \mathcal{I} \mathscr{E}_{\mathfrak{O}}\right)_{\mathfrak{Y}}$ is a $\{\mu\}$-filtration with respect to $\left(f^{*} t_{\alpha}\right)$. (Note that the conjugacy class $\{\mu\}$ has a cocharacter defined over some finite étale extension $R^{\prime}$ of $R$, so we may choose $\mathfrak{Y}$ to be an étale covering of $\mathfrak{X}_{R^{\prime}}$, which is also an étale covering of $\mathfrak{X}$.)

Note that $\Gamma(\mathfrak{X}, G)$ naturally acts on the set of $\{\mu\}$-filtrations. In practice (that is, when $\mathscr{E}$ comes from a suitable cohomology sheaf for an abelian scheme), it is too much to expect that $P_{\mathfrak{X}}$ is a trivial $G$-torsor - for example, $\mathscr{E}$ may not necessarily be a free $\mathcal{O}_{\mathfrak{X}}$-module. But it is certainly reasonable to impose that $P_{\mathfrak{X}}$ is a $G$-torsor; that is, that $\left(\mathscr{E},\left(t_{\alpha}\right)\right)$ étale-locally looks like $\left(M,\left(s_{\alpha}\right)\right)$.

When $G=\operatorname{GL}_{R}(M)$, then a filtration $\mathrm{Fil} \mathscr{E}$ of $\mathscr{E}$ is a $\{\mu\}$-filtration for some cocharacter $\mu$ if and only if associated grading $g r \bullet \mathscr{E}$ is of constant rank, and the conjugacy class of $\mu$ is uniquely determined by the rank of each grading.

Let us fix $G \subset \mathrm{GL}_{R}(M),\left(s_{\alpha}\right)$, and $\{\mu\}$ as in Definition 2.5.5, and consider a vector bundle $\mathscr{E}$ on $\mathfrak{X}$ and $\left(t_{\alpha}\right) \subset \Gamma(\mathfrak{X}, \mathscr{E} \otimes)$. Let $F 1_{G,\{\mu\}}^{\mathscr{E},\left(t_{\alpha}\right)}$ denote the functor on schemes on $\mathfrak{X}$, which associates to $\mathfrak{Y} \stackrel{f}{\rightarrow} \mathfrak{X}$ the set of $\{\mu\}$-filtration of $f^{*} \mathscr{E}$ with respect to $\left(f^{*} t_{\alpha}\right)$. We write $\mathrm{F} 1_{\{\mu\}}^{\mathscr{E}}:=\mathrm{F} 1_{\mathrm{GL}(M),\{\mu\}}^{\mathscr{E}, \emptyset}$, and we use the same letter to denote the scheme representing the functor, which is relative projective and smooth over $\mathfrak{X}$.

Lemma 2.5.6. The natural inclusion $\mathrm{F} 1_{G,\{\mu\}}^{\mathscr{E},\left(t_{\alpha}\right)} \hookrightarrow \mathrm{F}_{\{\mu\}}^{\mathscr{E}}$ can be represented by a closed immersion of schemes over $\mathfrak{X}$. Furthermore, if $P_{\mathfrak{X}}($ as in (2.5.4)) is a $G$ torsor, then $\mathrm{F}_{G,\{\mu\}}^{\mathscr{E},\left(t_{\alpha}\right)}$ is representable by a (nonempty) connected scheme which is relatively projective and smooth over $\mathfrak{X}$.

Proof. By étale descent of closed immersions, we may prove the lemma after replacing $R$ with some finite étale extension. So it suffices to show the lemma when $\{\mu\}$ contains a cocharacter defined over $R$, in which case the lemma was proved in [12, Lemma 2.2.6].

\subsection{Review on $\boldsymbol{G}$-isocrystals. We set $W:=W\left(\overline{\mathbb{F}}_{p}\right)$ and $K_{0}:=$ Frac $W$.}

Definition 2.6.1. Let $\mathbf{D}$ be a pro-torus with character group $X^{*}(\mathbf{D})=\mathbb{Q}$; that is, $\mathbf{D}=\lim \mathbb{G}_{m}$ where the transition maps is the $N$ th power maps ordered by divisibility.

For any morphism $v: \mathbf{D} \rightarrow \mathrm{GL}(n)_{K_{0}}$ we obtain a $\mathbb{Q}$-grading of $K_{0}^{n}$ by the weight decomposition. More explicitly, choose an integer $r$ such that $r v$ factors through $\mathbb{G}_{m}$. Then the $(d / r)$ th grading of $K_{0}^{n}$ is the subspace where the action of $(r v)(z)$ for $z \in \mathbb{G}_{m}\left(K_{0}\right)$ coincides with the scalar multiplication of $z^{d}$. 
Proposition 2.6.2 (Kottwitz [18]). Let $G$ be a connected reductive group over $\mathbb{Q}_{p}$. Then, for each $b \in G\left(K_{0}\right)$, there exists a unique homomorphism

$$
v_{b}: \mathbf{D} \rightarrow G_{K_{0}}
$$

such that for any representation $\rho: G_{K_{0}} \rightarrow \mathrm{GL}(n)_{K_{0}}$ the $\mathbb{Q}$-grading associated to $\rho \circ v_{b}$ is the slope decomposition for $\left(K_{0}^{n}, b \sigma\right)$. The $G\left(K_{0}\right)$-conjugacy class of $v_{b}$ only depends on the $\sigma$-conjugacy class of $b$ in $G\left(K_{0}\right)$.

Furthermore, any $\sigma$-conjugacy class of $G\left(K_{0}\right)$ contains an element $b \in G\left(K_{0}\right)$ which satisfy the following 'decency equation' for some $r \in \mathbb{Z}$ :

$$
(b \sigma)^{r}=\left(r v_{b}\right)(p) \sigma^{r}
$$

where the equality takes place in $\langle\sigma\rangle \ltimes G\left(K_{0}\right)$. (Recall that $r v_{b}$ factors through $\mathbb{G}_{m}$.)

It follows (from the uniqueness assertion) that for any $g, b \in G\left(K_{0}\right)$ we have $v_{g b \sigma(g)^{-1}}=g v_{b} g^{-1}$.

Consider the following group valued functor $J_{b}=J_{G, b}$ defined as follows:

$$
J_{b}(R):=\left\{g \in G\left(R \otimes_{\mathbb{Q}_{p}} K_{0}\right) \mid g b \sigma(g)^{-1}=b\right\}
$$

for any $\mathbb{Q}_{p}$-algebra $R$. Note that for any $g, b \in G\left(K_{0}\right)$ we have $J_{g b \sigma(g)^{-1}}(R)=$ $g J_{b}(R) g^{-1}$ as a subgroup of $G\left(R \otimes_{\mathbb{Q}_{p}} K_{0}\right)$; in particular, $J_{b}$ essentially depends only on the $\sigma$-conjugacy class of $b$ in $G\left(K_{0}\right)$.

Proposition 2.6.5. Assume that $b \in G\left(K_{0}\right)$ satisfies the decency equation (2.6.3) for $r \in \mathbb{Z}$. Then we have $\left(r v_{b}\right)(p) \in G\left(\mathbb{Q}_{p^{r}}\right) \cap J_{b}\left(\mathbb{Q}_{p}\right)$, where the intersection takes place in $G\left(K_{0}\right)$, and $J_{b}$ is representable by an inner form of the centralizer $G_{\left(r v_{b}\right)(p)}$, which is a Levi subgroup of $G$. (In particular, $\left(r v_{b}\right)(p)$ lies in the centre of $J_{b}\left(\mathbb{Q}_{p}\right)$.)

Proof. See [26, Corollaries 1.9, 1.14] for the proof.

2.7. Review of Dieudonné crystals. Let $\mathfrak{X}$ be a formal scheme over $\operatorname{Spf} \mathbb{Z}_{p}$, and consider the crystalline site $\left(\mathfrak{X} / \mathbb{Z}_{p}\right)$. By an isocrystal over $\mathfrak{X}$, we mean an object in the isogeny category of crystals of quasicoherent $\mathcal{O}_{\left(\mathfrak{X} / \mathbb{Z}_{p}\right)}$-modules. For any crystal of quasicoherent $\mathcal{O}_{\left(\mathfrak{X} / \mathbb{Z}_{p}\right)}$-modules $\mathbb{D}$, we let $\mathbb{D}\left[\frac{1}{p}\right]$ denote the associated isocrystal.

For a $p$-divisible group $X$ over $\mathfrak{X}$, we have a contravariant Dieudonné crystal $\mathbb{D}(X)$ equipped with a filtration $(\operatorname{Lie} X)^{*} \cong \mathrm{Fi} I_{X}^{1} \subset \mathbb{D}(X)_{\mathfrak{X}}$ by a subvector bundle, where $\mathbb{D}(X)_{\mathfrak{X}}$ is the pull-back of $\mathbb{D}(X)$ to the Zariski site of $\mathfrak{X}$. We call 
$F i I_{X}^{1}$ the Hodge filtration for $X$. If $\mathfrak{X}=\operatorname{Spf} R$, then we can regard the Hodge filtration as a filtration on the $R$-sections $\mathrm{Fil}_{X}^{1} \subset \mathbb{D}(X)(R)$. From the relative Frobenius morphism $F: X_{\overline{\mathfrak{X}}} \rightarrow \sigma^{*} X_{\bar{X}}$, we obtain the Frobenius morphism $F: \sigma^{*} \mathbb{D}(X) \rightarrow \mathbb{D}(X)$. On tensor products of $\mathbb{D}(X)$ 's, we naturally extend the Frobenius structure and filtration. (See [22], [21], or [1] for the construction of $\mathbb{D}(X)$ and the extra structure.)

If $X=\mathcal{A}\left[p^{\infty}\right]$ for some abelian scheme $f: \mathcal{A} \rightarrow \mathfrak{X}$, then we have $\mathbb{D}(X) \cong$ $R^{1} f_{\mathrm{CRIS}, *} \mathcal{O}_{\left(\mathcal{A} / \mathbb{Z}_{p}\right)}$, where the Frobenius morphism $F$ on $\mathbb{D}(X)$ matches with the crystalline Frobenius on the right hand side. Furthermore, restricting the isomorphism to the Zariski site, we obtain a filtered isomorphism between the vector bundle $\mathbb{D}(X)_{\mathfrak{X}}$ and the de Rham cohomology $\mathrm{H}_{\mathrm{dR}}^{1}(\mathcal{A} / \mathfrak{X})=\mathcal{V}(\mathcal{A})^{*}$ (both equipped with the Hodge filtration).

Let $\mathbf{1}:=\mathbb{D}\left(\mathbb{Q}_{p} / \mathbb{Z}_{p}\right)$ and $\mathbf{1}(-1):=\mathbb{D}\left(\mu_{p^{\infty}}\right)$. We set

- $\mathbf{1}(-c):=\mathbf{1}(-1)^{\otimes c}$ if $c>0$;

- $\mathbf{1}(-c):=\left(\mathbf{1}(-1)^{*}\right)^{\otimes|c|}$ if $c<0$;

- $\mathbf{1}(0):=\mathbf{1}$.

We often use the same notation $\mathbf{1}(-c)$ for the isocrystal associated to it. Note that the underlying crystal of $\mathbf{1}(-c)$ is the structure sheaf $\mathcal{O}_{\mathfrak{X} / \mathbb{Z}_{p}}$ with $F=p^{c}$ id, identifying $\sigma^{*} \mathbf{1}(-c)$ with $\mathcal{O}_{\mathfrak{X} / \mathbb{Z}_{p}}$ as well. (Note that such $F$ is only defined up to isogeny if $c<0$.) The Hodge filtration on $\mathbf{1}(-c)_{\mathfrak{X}}$ is concentrated at degree $c$.

Let $\mathcal{C}$ be the category of crystals of quasicoherent $\mathcal{O}_{\left(\mathfrak{X} / \mathbb{Z}_{p}\right)}$-modules, and $\mathcal{D} \subset \mathcal{C}$ be the full subcategory of finitely generated locally free objects. We now define $\mathbb{D}(X)^{\otimes} \in \mathcal{C}$ as in Section 2.2. Then the Hodge filtration on $\mathbb{D}(X)_{\mathfrak{X}}$ induces a natural filtration on $\mathbb{D}(X)_{\mathfrak{X}}^{\otimes}$, and the Frobenius morphism on $\mathbb{D}(X)$ induces an isomorphism of isocrystals $F: \sigma^{*} \mathbb{D}(X)^{\otimes}\left[\frac{1}{p}\right] \stackrel{\sim}{\rightarrow} \mathbb{D}(X)^{\otimes}\left[\frac{1}{p}\right]$.

DEFINITION 2.7.1. Let $X$ be a $p$-divisible group over a formal scheme $\mathfrak{X}$ over $\operatorname{Spf} \mathbb{Z}_{p}$. For a morphism of crystals $t: \mathbf{1} \rightarrow \mathbb{D}(X)^{\otimes}$, we let $t_{\mathrm{dR}}: \mathbf{1} \rightarrow \mathbb{D}(X)_{\mathfrak{X}}^{\otimes}$ denote the pull-back of $t$ to the Zariski site. By abuse of notation, we also denote by $t_{\mathrm{dR}} \in \Gamma\left(\mathfrak{X}, \mathbb{D}(X)_{\mathfrak{X}}^{\otimes}\right)$ the image of $1 \in \Gamma\left(\mathfrak{X}, \mathcal{O}_{\mathfrak{X}}\right)$ by $t_{\mathrm{dR}}$.

DEFINITION 2.7.2. Let $X$ be a $p$-divisible group over a formal scheme $\mathfrak{X}$ over $\operatorname{Spf} \mathbb{Z}_{p}$. A crystalline Tate tensor on $X$ is a morphism of crystals $t: \mathbf{1} \rightarrow \mathbb{D}(X)^{\otimes}$, which satisfies the following properties:

(1) The map on isocrystals $\mathbf{1} \rightarrow \mathbb{D}(X)^{\otimes}\left[\frac{1}{p}\right]$ induced $t$ is $F$-equivariant.

(2) The map $t_{\mathrm{dR}}$ is a de Rham tensor; that is, the section $t_{\mathrm{dR}} \in \Gamma\left(\mathfrak{X}, \mathbb{D}(X)_{\mathfrak{X}}^{\otimes}\right)$ lies in the 0 th filtration with respect to the filtration induced by $\mathrm{Fi}^{1}{ }_{X}^{1} \subset \mathbb{D}(X)_{\mathfrak{X}}$. 
Let $\mathfrak{X}$ be a formal scheme over $\operatorname{Spf} \mathbb{Z}_{p}$, and $f: \mathcal{A} \rightarrow \mathfrak{X}$ be an abelian scheme. Then a crystalline Tate tensor on $A$ is a morphism of crystals

$$
t: \mathbf{1} \rightarrow\left(R^{1} f_{\mathrm{CRIS}, *} \mathcal{O}_{\mathcal{A} / \mathbb{Z}_{p}}\right)^{\otimes}=\mathbb{D}\left(\mathcal{A}\left[p^{\infty}\right]\right)^{\otimes},
$$

which is a crystalline Tate tensor on $\mathcal{A}\left[p^{\infty}\right]$.

EXAMPLE 2.7.4. Given an endomorphism of $p$-divisible groups $f: X \rightarrow X$ we obtain a morphism of crystals $\mathbb{D}(f): \mathbb{D}(X) \rightarrow \mathbb{D}(X)$, which gives rise to the following crystalline Tate tensor:

$$
t_{f}: \mathbf{1} \rightarrow \mathbb{D}(X) \otimes \mathbb{D}(X)^{*} \subset \mathbb{D}(X)^{\otimes} .
$$

To a principal polarization $\lambda: X \stackrel{\sim}{\rightarrow} X^{\vee}$ one can associate a crystalline Tate tensor $t_{\lambda}: 1 \rightarrow \mathbb{D}(X)^{\otimes}$ by the same recipe as in [12, Example 2.1.4].

\section{Review on Shimura varieties of Hodge type}

We review basic results on Shimura varieties of Hodge type and their integral models in the good reduction case. Our notation is a global analogue of the notation introduced in [12, Section 2]. In Section 4 we recall the main results of [12].

\subsection{Review of Shimura varieties of Hodge type in characteristic 0 .} Consider a $2 g$-dimensional $\mathbb{Q}$-vector space $V$, equipped with a nondegenerate alternating bilinear form (that is, a symplectic form) $\psi: V \times V \rightarrow \mathbb{Q}$. Consider the symplectic similitude group $\operatorname{GSp}(V, \psi)$ which is a connected reductive group. One can find an $\mathbb{R}$-basis of $V_{\mathbb{R}}$ so that the matrix representation of

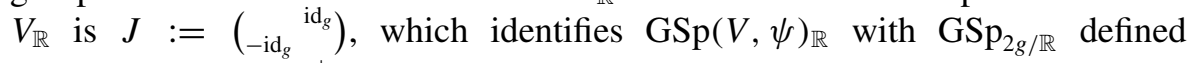
by $\left(\mathbb{R}^{2 g}, J\right)$. Let $\mathcal{S}^{ \pm}$be the set of $\mathrm{GSp}_{2 g}(\mathbb{R})$-conjugates of the cocharacter $h: \operatorname{Res}_{\mathbb{C} / \mathbb{R}} \mathbb{G}_{m} \rightarrow \mathrm{GSp}_{2 g / \mathbb{R}}$ which induces the following on the $\mathbb{R}$-points:

$$
\mathbb{C}^{\times} \rightarrow \mathrm{GSp}_{2 g}(\mathbb{R}) ; \quad a+b i \mapsto\left(\begin{array}{cc}
a \mathrm{id}_{g} & b \mathrm{id}_{g} \\
-b \mathrm{id}_{g} & a \mathrm{id}_{g}
\end{array}\right) .
$$

Then $\left(\operatorname{GSp}(V, \psi), \mathcal{S}^{ \pm}\right)$is a Shimura datum, often referred to as a Siegel Shimura datum. Its reflex field is $\mathbb{Q}$.

Definition 3.1.1. A Shimura datum $(G, \mathfrak{H})$ is called of Hodge type if there is an embedding of Shimura data

$$
(G, \mathfrak{H}) \hookrightarrow\left(\mathrm{GSp}(V, \psi), \mathcal{S}^{ \pm}\right)
$$

for some rational symplectic vector space $(V, \psi)$. 
Clearly, $\left(\operatorname{GSp}(V, \psi), \mathcal{S}^{ \pm}\right)$is of Hodge type. More generally, PEL-type Shimura data (cf. [6, Section 4], [19, Section 4]) are of Hodge type.

3.1.2. Let $(G, \mathfrak{H})$ be a Shimura datum of Hodge type. To simplify the notation, let $E:=E(G, \mathfrak{H})$ denote the reflex field and we write $\operatorname{Sh}_{\mathrm{K}}:=\operatorname{Sh}_{\mathrm{K}}(G, \mathfrak{H})$ to denote the canonical model over $E$. We fix an embedding $(G, \mathfrak{H}) \hookrightarrow\left(\operatorname{GSp}(V, \psi), \mathcal{S}^{ \pm}\right)$, and let $\mathrm{K} \subset G\left(\mathbb{A}_{\mathrm{f}}\right)$ and $\mathrm{K}^{\prime} \subset \mathrm{GSp}(V, \psi)\left(\mathbb{A}_{\mathrm{f}}\right)$ be 'small enough' open compact subgroups with $\mathrm{K} \subset \mathrm{K}^{\prime}$ such that the natural map $\operatorname{Sh}_{\mathrm{K}}(G, \mathfrak{H}) \rightarrow \operatorname{Sh}_{\mathrm{K}^{\prime}}(\mathrm{GSp}(V$, $\left.\psi), \mathcal{S}^{ \pm}\right)_{E(G, \mathfrak{H})}$ is a closed immersion. (Indeed, up to replacing $\mathrm{K} \subset G\left(\mathbb{A}_{\mathrm{f}}\right)$ with some finite-index open subgroup it is always possible to find $K^{\prime}$ as above; cf. [6, Proposition 1.15].)

Recall that $\operatorname{Sh}_{\mathrm{K}^{\prime}}\left(\mathrm{GSp}(V, \psi), \mathcal{S}^{ \pm}\right)$can be interpreted as a moduli space of polarized complex abelian varieties with level structure, so we have a universal abelian scheme $\mathcal{A}_{\mathrm{K}^{\prime}, \mathbb{Q}} \rightarrow \operatorname{Sh}_{\mathrm{K}^{\prime}}\left(\mathrm{GSp}(V, \psi), \mathcal{S}^{ \pm}\right)$defined up to isogeny. By restriction, we obtain an abelian scheme $f: \mathcal{A}_{K, E} \rightarrow \mathrm{Sh}_{\mathrm{K}}(G, \mathfrak{H})$. Pulling back by $E \hookrightarrow \mathbb{C}$, we can explicitly write down a 'universal abelian scheme (up to isogeny)' $f: \mathcal{A}_{\mathrm{K}, \mathbb{C}} \rightarrow \mathrm{Sh}_{\mathrm{K}}(G, \mathfrak{H})_{\mathbb{C}}$ in terms of the associated variation of $\mathbb{Q}$ Hodge structures. First, the first Betti homology can be obtained as follows:

$$
\mathcal{V}\left(\mathcal{A}_{\mathrm{K}, \mathbb{C}}\right)=G(\mathbb{Q}) \backslash\left(V \times \mathfrak{H} \times G\left(\mathbb{A}_{\mathrm{f}}\right)\right) / \mathrm{K}, \quad \text { cf. (2.4.3), }
$$

where $G(\mathbb{Q})$ acts diagonally and $\mathrm{K}$ acts only on $G\left(\mathbb{A}_{\mathrm{f}}\right)$. To define the Hodge filtration, consider the following filtration $\mathrm{Fil}_{\mathfrak{H}}^{\bullet}$ of $V \times \mathfrak{H}$ whose fibre at $h \in \mathfrak{H}$ is given by grading induced from the cocharacter $\mu_{h}: \mathbb{G}_{m} \rightarrow G_{\mathbb{C}}$ (in the sense of Definition 2.5.3), where $\mu_{h}$ is as below:

$$
\mu_{h}: \mathbb{G}_{m} \stackrel{z \mapsto(z, 1)}{\longrightarrow} \mathbb{G}_{m} \times \mathbb{G}_{m} \cong \mathbb{S}_{\mathbb{C}} \stackrel{h_{\mathbb{C}}}{\longrightarrow} G_{\mathbb{C}}
$$

Then $\mathrm{Fil}_{\mathfrak{H}}^{\bullet}$ descends to a holomorphic filtration $\mathrm{Fi} I_{\mathrm{K}}^{\bullet}$ of $\mathcal{O}_{\mathrm{Sh}_{K}, \mathbb{C}} \otimes_{\mathbb{Q}} \mathcal{V}\left(\mathcal{A}_{\mathrm{K}, \mathbb{C}}\right)$. This define a variation of $\mathbb{Q}$-Hodge structures that defines $\mathcal{A}_{\mathrm{K}, \mathbb{C}}$.

Lemma 3.1.5. Let $s \in V^{\otimes}$ be an element fixed by $G$. Then the morphism $\mathbf{1} \rightarrow V^{\otimes}$ defined by $1 \mapsto$ s induce a morphism $t_{B}^{\text {univ }}: \mathbf{1} \rightarrow \mathcal{V}\left(\mathcal{A}_{\mathrm{K}, \mathbb{C}}\right)^{\otimes}$ of 'variations of $\mathbb{Q}$-Hodge structures'. Furthermore, such $t_{B}^{\text {univ }}$ is compatible under the natural

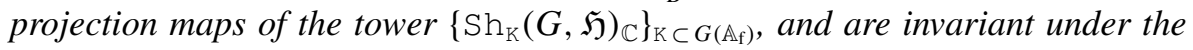
natural $G\left(\mathbb{A}_{\mathrm{f}}\right)$-action.

Proof. If $s$ is fixed by $G$, then the global section $s \in \Gamma\left(\mathfrak{H}, V^{\otimes} \times \mathfrak{H}\right)$ induce a global section $s$ of $\mathcal{V}\left(\mathcal{A}_{\mathrm{K}, \mathbb{C}}\right)$. Therefore we obtain a $\mathbb{Q}$-linear morphism of locally constant sheaves $t_{B}^{\text {univ }}: \mathbf{1} \rightarrow \mathcal{V}\left(\mathcal{A}_{\mathrm{K}, \mathbb{C}}\right)^{\otimes}$. To show that the image of this map is in the 0th filtration, it suffices to show the claim over $\mathfrak{H}$, but by definition of the filtration $\mathrm{Fi} I_{\mathfrak{H}}^{\bullet}$ the global section $s$ has to lie in the 0th filtration (as $s$ is fixed by $G$ ). 
The last assertion (on the compatibility with the tower and the Hecke $G\left(\mathbb{A}_{\mathrm{f}}\right)$ action) is clear.

Lemma 3.1.6. Let $s \in V^{\otimes}$ be an element fixed by $G$, and $t_{B}^{\text {univ }}: \mathbf{1} \rightarrow \mathcal{V}\left(\mathcal{A}_{\mathrm{K}, \mathbb{C}}\right)^{\otimes}$ be the morphism constructed from s by the recipe in Lemma 3.1.5. Then the de Rham component $t_{\mathrm{dR}}^{\text {univ }}: \mathbf{1} \rightarrow \mathcal{V}_{\mathrm{dR}}\left(\mathcal{A}_{\mathrm{K}, \mathbb{C}}\right)^{\otimes}$ of $t_{B}^{\text {univ }}(c f .(2.4 .4 b))$ descends to a de Rham tensor $t_{\mathrm{dR}}^{\text {univ }}: \mathbf{1} \rightarrow \mathcal{V}_{\mathrm{dR}}\left(\mathcal{A}_{\mathrm{K}, E}\right)^{\otimes}$, and the étale component $t_{\mathrm{et}}^{\text {univ }}: \mathbf{1} \rightarrow \mathcal{V}_{\mathbb{A}_{\mathrm{f}}}\left(\mathcal{A}_{\mathrm{K}, \mathbb{C}}\right)^{\otimes}$ of $t_{B}^{\text {univ }}(c f .(2.4 .4 a))$ descends to an étale tensor $t_{\mathrm{et}}^{\text {univ }}: \mathbf{1} \rightarrow \mathcal{V}_{\mathbb{A}_{\mathrm{f}}}\left(\mathcal{A}_{\mathrm{K}, E}\right)^{\otimes}$.

Proof. This lemma is essentially proved in Lemma 2.2.1 and Corollary 2.2.2 in [14], by choosing a finitely many tensors $\left(s_{\alpha}\right) \subset V^{\otimes}$ such that their pointwise stabilizer is $G$ and one of $s_{\alpha}$ is $s$. We now explain how to deduce the lemma from [14].

The existence of the de Rham tensor $t_{\mathrm{dR}}^{\text {univ }}$ on $\mathcal{A}_{\mathrm{K}, E}$ is proved in [14, Corollary 2.2.2]. Let us now prove the assertion on the étale components. Let $\eta$ be a generic point of $\mathrm{Sh}_{\mathrm{K}}$, and $\bar{\eta}$ be a geometric point supported at $\eta$. By [14, Lemma 2.2.1], the fibre $t_{\mathrm{et}, \bar{\eta}}^{\text {univ }}$ is invariant under the action of $\operatorname{Gal}(\bar{\eta} / \eta)$.

Let $\overline{\{\eta\}} \subset \mathrm{Sh}_{\mathrm{K}}$ be the connected component of $\mathrm{Sh}_{\mathrm{K}}$ containing $\eta$. Since $\pi_{1}^{\text {ét }}(\overline{\{\eta\}}, \bar{\eta})$ is a quotient of $\operatorname{Gal}(\bar{\eta} / \eta)$ by normality, it follows $t_{\mathrm{et}, \eta}^{\text {univ }}$ extends over $\overline{\{\eta\}}$. Hence we obtain $t_{\mathrm{et}}^{\text {univ }}$ over $S h_{\mathrm{K}}$ by repeating this process for each of the generic points.

\section{2. 'Universal' abelian schemes over Hodge-type Shimura varieties (in} characteristic 0). We fix finitely many elements $\left(s_{\alpha}\right) \subset V^{\otimes}$ whose pointwise stabilizer is $G \subset \mathrm{GL}_{\mathbb{Q}}(V)$; cf. Proposition 2.5.1. Consider $t_{\alpha, B}^{\text {univ }}: \mathbf{1} \rightarrow \mathcal{V}\left(\mathcal{A}_{\mathrm{K}, \mathbb{C}}\right)^{\otimes}$ associated to $\left(s_{\alpha}\right)$ by Lemma 3.1.5, which produce $t_{\alpha, \mathrm{dR}}^{\text {univ }}$ and $t_{\alpha, \text { ét }}^{\text {univ }}$ defined over $S \mathrm{~S}_{\mathrm{K}}$ by Lemma 3.1.6. We now list the properties and extra structures possessed by $\mathcal{A}_{\mathrm{K}, E}$.

3.2.1 ( $G$-torsor). Consider the following closed subscheme of the isom scheme over $\mathrm{Sh}_{\mathrm{K}}$

$$
P_{\mathrm{K}, E} \subset \operatorname{isom}_{\mathrm{Sh}_{\mathrm{K}}}\left(\mathcal{V}_{\mathrm{dR}}\left(\mathcal{A}_{\mathrm{K}, E}\right), \mathcal{O}_{\mathrm{Sh}_{\mathrm{K}}} \otimes_{\mathbb{Q}} V\right),
$$

defined by the condition of matching $\left(t_{\alpha, \mathrm{dR}}^{\text {univ }}\right) \subset \Gamma\left(\mathrm{Sh}_{\mathrm{K}}, \mathcal{V}_{\mathrm{dR}}\left(\mathcal{A}_{\mathrm{K}, E}\right)^{\otimes}\right)$ with $\left(s_{\alpha}\right) \subset V^{\otimes}$ for each $\alpha$. Then $P_{\mathrm{K}, E}$ is a $G$-torsor. Indeed, it suffices to show that $P_{\mathrm{K}, \mathbb{C}}$ is a $G$-torsor over $\mathrm{Sh}_{\mathrm{K}, \mathbb{C}}$. On the other hand, $P_{\mathrm{K}, \mathbb{C}}$ splits under the complex analytic topology (which can be seen from the natural isomorphism $\mathcal{V}_{\mathrm{dR}}\left(\mathcal{A}_{\mathrm{K}, \mathbb{C}}\right) \cong \mathcal{O}_{\mathrm{Sh}_{\mathrm{K}, \mathbb{C}}} \otimes_{\mathbb{Q}} \mathcal{V}\left(\mathcal{A}_{\mathrm{K}, \mathbb{C}}\right)$ and the explicit construction of $\left.\mathcal{V}\left(\mathcal{A}_{\mathrm{K}, \mathbb{C}}\right)\right)$, so $P_{\mathrm{K}, \mathbb{C}} \rightarrow \mathrm{Sh}_{\mathrm{K}, \mathbb{C}}$ is flat with nonempty fibre everywhere and the natural $G$-action is simple and transitive. 
3.2.2 (The Hodge filtration is a $\{\mu\}$-filtration.). Recall that the conjugacy class $\{\mu\}$ of $\mu_{h}$ associated to some $h \in \mathfrak{H}$ by (3.1.4) is independent of $h$ and is defined over $E=E(G, \mathfrak{H})$. Then the Hodge filtration $\mathrm{Fil}^{0}\left(\mathcal{V}_{\mathrm{dR}}\left(\mathcal{A}_{\mathrm{K}, E}\right)\right)$ is a $\{\mu\}$-filtration with respect to $\left(t_{\alpha, \mathrm{dR}}^{\mathrm{univ}}\right)$ in the sense of Definition 2.5.5. Indeed, since $S h_{\mathrm{K}}$ is reduced and of finite type, it suffices (by Lemma 2.5.6) to show that at each closed point $x \in \mathrm{Sh}_{\mathrm{K}}(\mathbb{C})$ the fibre

$$
\operatorname{Fil}{ }^{0}\left(\mathcal{V}_{\mathrm{K}, x}\right) \subset \mathcal{V}_{\mathrm{dR}}\left(\mathcal{A}_{\mathrm{K}, E}\right)_{x} \cong \mathrm{H}_{\mathrm{dR}}^{1}\left(\mathcal{A}_{\mathrm{K}, x} / \mathbb{C}\right)^{*}
$$

defines a point in $\mathrm{F}_{G,\{\mu\}}^{\mathcal{V}_{\mathrm{dR}}\left(\mathcal{A}_{K, E}\right),\left(t_{\alpha, \mathrm{dR}}^{\text {univ }}\right)}$ over $x \in \mathrm{Sh}_{K}(\mathbb{C})$. And this is clear from the definition, as the Hodge filtration at $x$ is given by the cocharacter $\mu_{h}$ associated to some $h \in \mathfrak{H}$.

3.2.3 (Level Structure). For an open compact subgroup $\mathrm{K} \subset G\left(\mathbb{A}_{\mathrm{f}}\right)$, we define a universal global section

$$
\eta_{\mathrm{K}} \in \Gamma\left(\operatorname{Sh}_{\mathrm{K}}, \operatorname{isom}\left[\left(V_{\mathbb{A}_{\mathrm{f}}},\left(1 \otimes s_{\alpha}\right)\right),\left(\mathcal{V}_{\mathbb{A}_{\mathrm{f}}}\left(\mathcal{A}_{\mathrm{K}, E}\right),\left(t_{\alpha, \text { ét }}^{\text {univ }}\right)\right)\right] / \mathrm{K}\right),
$$

where $V_{\mathbb{A}_{\mathrm{f}}}:=\mathbb{A}_{\mathrm{f}} \otimes_{\mathbb{Q}} V$. Note that $\eta_{K}$ only depends on the isogeny class of $\left(\mathcal{A}_{\mathrm{K}, E}\right.$, $\left.\left(t_{\alpha, \text { ét }}^{\text {univ }}\right)\right)$; that is, $\mathcal{A}_{\mathrm{K}, E}$ up to isogeny respecting $\left(t_{\alpha, \text { ét }}^{\text {univ }}\right)$.

For a geometric point $x \in \mathrm{Sh}_{\mathrm{K}}(\mathbb{C})$, let $\pi_{1}^{\text {et }}\left(\mathrm{Sh}_{\mathrm{K}}, x\right)$ and $\pi_{1}^{\text {ét }}\left(\mathrm{Sh}_{\mathrm{K}, \mathbb{C}}, x\right)$ denote the étale fundamental group of the component containing $x$. Then defining $\eta_{\mathrm{K}}$ is equivalent to giving, for a point $x \in \mathrm{Sh}_{\mathrm{K}}(\mathbb{C})$ on each connected component, an isomorphism

$$
\eta_{x}: V_{\mathbb{A}_{\mathrm{f}}} \stackrel{\sim}{\rightarrow} \mathcal{V}_{\mathbb{A}_{\mathrm{f}}}\left(\mathcal{A}_{\mathrm{K}, E}\right)_{x},
$$

matching $\left(1 \otimes s_{\alpha}\right)$ and $\left(t_{\alpha, \text { ét, } x}^{\text {univ }}\right)$, such that the right coset $\eta_{x} \mathrm{~K}$ is stable under the action of $\pi_{1}^{\text {et }}\left(\mathrm{Sh}_{\mathrm{K}}, x\right)$.

Note that the pull-back of $\mathcal{V}_{\mathrm{K}}$ to $\mathfrak{H} \times G\left(\mathbb{A}_{\mathrm{f}}\right)$ is a trivial local system. We first define $\tilde{\eta}: V_{\mathbb{A}_{\mathrm{f}}} \times \mathfrak{H} \times G\left(\mathbb{A}_{\mathrm{f}}\right) \rightarrow V_{\mathbb{A}_{\mathrm{f}}} \times \mathfrak{H} \times G\left(\mathbb{A}_{\mathrm{f}}\right)$ by $(v, h, g) \mapsto(g v, h, g)$. Given a point $x \in \mathrm{Sh}_{\mathrm{K}}(\mathbb{C})$, we pick a lift $(h, g) \in \mathfrak{H} \times G\left(\mathbb{A}_{\mathrm{f}}\right)$ of $x$ and set

$$
\eta_{x}:=\left.\tilde{\eta}\right|_{V_{\mathbb{A}_{\mathrm{f}}} \times\{(h, g)\}}: V_{\mathbb{A}_{\mathrm{f}}} \rightarrow V_{\mathbb{A}_{\mathrm{f}}} .
$$

We now show that the right coset $\eta_{x} \mathrm{~K}$ only depends on $x$, not on the choice of lift $(h, g)$, where $g^{\prime} \in \mathrm{K}$ acts as $\eta_{x} \mapsto \eta_{x} \circ g^{\prime}$.

Firstly, for any $g^{\prime} \in \mathrm{K}$ we obtain another lift $\left(h, g g^{\prime}\right) \in \mathfrak{H} \times G\left(\mathbb{A}_{\mathrm{f}}\right)$ of $x$. Then we have

$$
\widetilde{\eta}\left(v, h, g g^{\prime} \mathrm{K}\right)=\left(g g^{\prime} v, h, g g^{\prime} \mathrm{K}\right)=\widetilde{\eta}\left(g^{\prime} v, h, g \mathrm{~K}\right) \in V_{\mathbb{A}_{\mathrm{f}}} \times \mathfrak{H} \times G\left(\mathbb{A}_{\mathrm{f}}\right) / \mathrm{K}
$$

that is, we have $\tilde{\eta}_{\left(h, g g^{\prime} \mathrm{K}\right)}=\eta_{(h, g \mathrm{~K})} \circ g^{\prime}$. 
Secondly, for any $\gamma \in G(\mathbb{Q})$ we obtain another lift $\left({ }^{\gamma} h, \gamma g\right) \in \mathfrak{H} \times G\left(\mathbb{A}_{\mathrm{f}}\right)$ of $x$. Then we have

$$
\tilde{\eta}\left(v,{ }^{\gamma} h, \gamma g \mathrm{~K}\right)=\left(\gamma g v,{ }^{\gamma} h, \gamma g \mathrm{~K}\right)=(\gamma, \gamma, \gamma) \circ \widetilde{\eta}(v, h, g \mathrm{~K}) .
$$

Now, recall that $\mathcal{V}_{\mathrm{K}}=G(\mathbb{Q}) \backslash\left(V \times \mathfrak{H} \times G\left(\mathbb{A}_{\mathrm{f}}\right)\right) / \mathrm{K}$ where $G(\mathbb{Q})$ acts diagonally. Therefore, we obtain the same map $\eta_{x}$ if we replace $(h, g)$ with $\left({ }^{\gamma} h, \gamma g\right)$.

This shows that the right coset $\eta_{x} \mathrm{~K}$ is stable under the action of $\pi_{1}^{\text {et }}\left(\mathrm{Sh}_{\mathrm{K}, \mathbb{C}}, x\right)$. We now show that $\eta_{x} \mathrm{~K}$ is stable under the action of $\pi_{1}^{\text {et }}\left(\mathrm{Sh}_{\mathrm{K}}, x\right)$. Clearly, we may replace $K$ with a finite-index open normal subgroup, so we may assume that there exists a 'small enough' open compact subgroup $\mathrm{K}^{\prime} \subset \mathrm{GSp}(V, \psi)\left(\mathbb{A}_{\mathrm{f}}\right)$ containing $\mathrm{K}$ such that $\mathrm{Sh}_{\mathrm{K}} \rightarrow \operatorname{Sh}_{\mathrm{K}^{\prime}}\left(\mathrm{GSp}(V, \psi), \mathcal{S}^{ \pm}\right)_{E}$ is a closed immersion. Then $\eta_{x} \mathrm{~K}^{\prime}$ defines a universal level structure on $\mathcal{A}_{\mathrm{K}^{\prime}, \mathbb{C}}$, so it 'descents' to a level structure on $\mathcal{A}_{\mathrm{K}^{\prime}, E}$ (by the universal property of $\mathcal{A}_{\mathrm{K}^{\prime}, E}$ ). In particular, $\eta_{x} \mathrm{~K}^{\prime}$ is stable under the action of $\pi_{1}^{\text {et }}\left(\mathrm{Sh}_{\mathrm{K}}, x\right)$. But since $\mathrm{Sh}_{\mathrm{K}}(\mathbb{C}) \rightarrow \operatorname{Sh}_{\mathrm{K}^{\prime}}\left(\mathrm{GSp}(V, \psi), \mathcal{S}^{ \pm}\right)(\mathbb{C})$ is injective, $\eta_{x} \mathrm{~K}$ is the only right $\mathrm{K}$-coset contained in $\eta_{x} \mathrm{~K}^{\prime}$ whose elements match $\left(1 \otimes s_{\alpha}\right)$ and $\left(t_{\alpha, \text { ét } x}^{\text {univ }}\right)$. (Indeed, if there were any other $\mathrm{K}$-coset $\eta_{y} \mathrm{~K} \subset \eta_{x} \mathrm{~K}^{\prime}$ with this property, then $\eta_{x} \mathrm{~K}$ and $\eta_{y} \mathrm{~K}$ define $\mathbb{C}$-points of $\mathrm{Sh}_{\mathrm{K}}$ which map to the same point in $\operatorname{Sh}_{\mathrm{K}^{\prime}}\left(\mathrm{GSp}(V, \psi), \mathcal{S}^{ \pm}\right)$.) Since $\left(t_{\alpha, \text { êt }, x}^{\text {univ }}\right)$ are invariant under the action of $\pi_{1}^{\text {ét }}\left(\mathrm{Sh}_{\mathrm{K}}, x\right)$ by Lemma 3.1.6, it also follows that $\eta_{x} \mathrm{~K}$ is stable under the action of $\pi_{1}^{\text {ét }}\left(\mathrm{Sh}_{\mathrm{K}}, x\right)$.

3.2.4 (Hecke action). For any $\mathrm{K} \subset G\left(\mathbb{A}_{\mathrm{f}}\right)$, the right translation by $g \in G\left(\mathbb{A}_{\mathrm{f}}\right)$ on $\mathfrak{H} \times G\left(\mathbb{A}_{\mathrm{f}}\right)$ descends to an isomorphism

$$
[g]: \mathrm{Sh}_{g \mathrm{Kg}}-1, \mathbb{C} \stackrel{\sim}{\rightarrow} \mathrm{Sh}_{\mathrm{K}, \mathbb{C}} .
$$

By the standard rigidity result (cf. [24, Theorem 13.6]), this map is defined over the reflex field

$$
[g]: \mathrm{Sh}_{g \mathrm{~K} g}{ }^{-1} \stackrel{\sim}{\rightarrow} \mathrm{Sh}_{\mathrm{K}} .
$$

We can describe the pull-back by $[g]$ of the universal abelian scheme and the level structure $\left(\mathcal{A}_{\mathrm{K}}, \eta_{\mathrm{K}}\right)$ as follows. The isogeny class of $[g]^{*}\left(\mathcal{A}_{\mathrm{K}},\left(t_{\alpha, \text { ét }}^{\text {univ }}\right)\right)$ coincides with $\left(\mathcal{A}_{g \mathrm{~K} g^{-1}},\left(t_{\alpha, \mathrm{et}}^{\text {univ }}\right)\right)$, and $[g]^{*} \eta_{\mathrm{K}}$ corresponds to $\left(\eta_{x}^{\prime} g\right) \mathrm{K}$ for any $x \in \mathrm{Sh}_{g \mathrm{~K} g^{-1}}(\mathbb{C})$ where $\eta_{x}^{\prime}: V_{\mathbb{A}_{\mathrm{f}}} \stackrel{\sim}{\rightarrow} \mathcal{V}_{\mathbb{A}_{\mathrm{f}}}\left(\mathcal{A}_{g \mathrm{~K} g^{-1}}\right)_{x}$ is a representative of the fibre of $\eta_{g \mathrm{~K} g^{-1}}$ at $x$. (These claims can be explicitly verified over $\mathbb{C}$.)

3.3. Integral canonical models. In this section, we review the basic properties of integral canonical models of Hodge-type Shimura varieties in the good reduction case, constructed independently by Kisin [14] and Vasiu [27-29]. We refer to the aforementioned references for the full details including the definition of integral canonical model. 
3.3.1 (Good Reduction Hypothesis). From now on, we fix a prime $p$. Let $(G, \mathfrak{H})$ be a Hodge-type Shimura datum, and assume that $G$ admits a reductive $\mathbb{Z}_{(p)^{-}}$ model $G_{\mathbb{Z}_{(p)}}$. Then we can choose the following extra data:

(1) We choose an embedding of Shimura data $(G, \mathfrak{H}) \hookrightarrow\left(\operatorname{GSp}(V, \psi), \mathcal{S}^{ \pm}\right)$, and a $\psi$-stable $\mathbb{Z}_{(p)}$-lattice $\Lambda_{\mathbb{Z}_{(p)}} \subset V$ such that the closed immersion $G \hookrightarrow$ $\operatorname{GSp}(V, \psi) \hookrightarrow \operatorname{GL}(V)$ over $\mathbb{Q}$ extends to a closed immersion $G_{\mathbb{Z}_{(p)}} \hookrightarrow$ $\operatorname{GL}\left(\Lambda_{\mathbb{Z}_{(p)}}\right)$ of reductive groups over $\mathbb{Z}_{(p)}$. If $p>2$ then for any embedding $(G, \mathfrak{H}) \hookrightarrow\left(\operatorname{GSp}(V, \psi), \mathcal{S}^{ \pm}\right)$there exists a lattice $\Lambda_{\mathbb{Z}_{(p)}}$ with the above property by [14, Proposition 2.3.1]. (Indeed, [14] shows that for any $G\left(\mathbb{Z}_{p}\right)$ stable $\mathbb{Z}_{p}$-lattice $\Lambda \subset \mathbb{Q}_{p} \otimes_{\mathbb{Q}} V, \Lambda_{\mathbb{Z}_{(p)}}:=\Lambda \cap V$ satisfies the desired property, unless $p=2$ and $G_{\overline{\mathbb{Q}}}$ has no normal subgroup isomorphic to $\mathrm{SO}_{2 n+1}$.)

(2) We choose finitely many elements $\left(s_{\alpha}\right) \subset \Lambda_{\mathbb{Z}_{(p)}}^{\otimes}$ such that the pointwise stabilizer of $\left(s_{\alpha}\right)$ in $\operatorname{GL}\left(\Lambda_{\mathbb{Z}_{(p)}}\right)$ is $G_{\mathbb{Z}_{(p)}}$, which is possible by Proposition 2.5.1.

We do not require $\psi$ to be a perfect alternating form on $\Lambda_{\mathbb{Z}_{(p)}}$, although by Zarhin's trick it is possible to arrange $(V, \psi)$ so that $\psi$ induces a perfect alternating form on some choice of $\Lambda_{\mathbb{Z}_{(p)}}$.

The following lemma is proved in [23, Corollary 4.7]:

Lemma 3.3.2. Let $(G, \mathfrak{H})$ be any Shimura datum. Assume that $G$ is unramified at $p$; that is, there exists a reductive $\mathbb{Z}_{(p)}$-model of $G$. Then the reflex field $E(G, \mathfrak{H})$ is unramified at any prime $\mathfrak{p}$ over $p$.

Recall that we fix a reductive $\mathbb{Z}_{(p)}$-model of $G$, also denoted by $G$, which is a closed subgroup of $\operatorname{GL}\left(\Lambda_{\mathbb{Z}_{(p)}}\right)$. Set $\mathrm{K}_{p}:=G\left(\mathbb{Z}_{p}\right) \subset G\left(\mathbb{Q}_{p}\right)$, which is a hyperspecial maximal compact subgroup. Choose an open compact subgroup $\mathrm{K}^{p} \subset G\left(\mathbb{A}_{\mathrm{f}}^{p}\right)$ so that the product $\mathrm{K}_{p} \mathrm{~K}^{p} \subset G\left(\mathbb{A}_{\mathrm{f}}\right)$ is 'small enough'. From now on, we always assume that $\mathrm{K}:=\mathrm{K}_{p} \mathrm{~K}^{p}$ with the hyperspecial maximal compact subgroup $\mathrm{K}_{p}$, in which case we expect that $\mathrm{Sh}_{\mathrm{K}}$ should admit a smooth integral model over $\mathscr{O}_{E,(\mathfrak{p})}$ for any prime $\mathfrak{p}$ of $E:=E(G, \mathfrak{H})$ over $p$. Here, $\mathscr{O}_{E,(\mathfrak{p})}$ is the (uncompleted) localization of $\mathscr{O}_{E}$ at $\mathfrak{p}$, which is an unramified extension of $\mathbb{Z}_{(p)}$ by Lemma 3.3.2.

Let us recall the main result and basic properties on integral canonical models:

Theorem 3.3.3 (Vasiu, Kisin). Assume that $p>2$. Then for any small enough $\mathrm{K}^{p}$ there exists an integral canonical $\mathscr{O}_{E,(\mathfrak{p})}$-model $\mathscr{S}_{\mathrm{K}_{p} \mathrm{~K}^{p}}$ of $\mathrm{S}_{\mathrm{K}_{p} \mathrm{~K}^{p}}$ in the following sense: The $G\left(\mathbb{A}_{\mathrm{f}}^{p}\right)$-equivariant tower $\left\{\mathrm{Sh}_{\mathrm{K}_{p} \mathrm{~K}^{p}}\right\}_{\mathrm{K}^{p}}$ extends to a $G\left(\mathbb{A}_{\mathrm{f}}^{p}\right)$-equivariant tower $\left\{\mathscr{S}_{\mathrm{K}_{p} K^{p}}\right\}_{K^{p}}$ of smooth $\mathscr{O}_{E,(\mathfrak{p})}$-schemes with finite étale transition maps, and 
the tower satisfies the (uniquely characterizing) extension property formulated by Milne (cf. [25, Section 3]).

Furthermore, the following additional properties hold:

(1) Choose a $\psi$-stable $\mathbb{Z}$-lattice $\Lambda_{\mathbb{Z}} \subset \Lambda_{\mathbb{Z}_{(p)}}$. Then the universal abelian scheme $\mathcal{A}_{\mathrm{K}_{p} \mathrm{~K}^{p}, E} \rightarrow \mathrm{Sh}_{\mathrm{K}_{p} \mathrm{~K}^{p}}$, corresponding to the choice of $\mathbb{Z}$-lattice $\Lambda_{\mathbb{Z}}$, extends to an abelian scheme $\mathcal{A}_{\mathrm{K}_{p} \mathrm{~K}^{p}} \rightarrow \mathscr{S}_{\mathrm{K}_{p} \mathrm{~K}^{p}}$.

(2) The de Rham tensors $\left(t_{\alpha, \mathrm{dR}}^{\mathrm{univ}}\right)$ on $\mathcal{A}_{\mathrm{K}_{p} \mathrm{~K}^{p}, E}$, associated to $\left(s_{\alpha}\right) \subset \Lambda_{\mathbb{Z}_{(p)}}^{\otimes}$ by Lemma 3.1.6, extends over the integral canonical model $t_{\alpha, \mathrm{dR}}^{\mathrm{univ}}: \mathbf{1} \rightarrow$ $\mathcal{V}_{\mathrm{dR}}\left(\mathcal{A}_{\mathrm{K}_{p} \mathrm{~K}^{p}}\right)^{\otimes}$. Furthermore, the formation of $t_{\alpha, \mathrm{dR}}^{\mathrm{univ}}$ respects the natural projections and the natural $G\left(\mathbb{A}_{\mathrm{f}}^{p}\right)$-action on the tower $\left\{\mathscr{S}_{\mathrm{K}_{p} \mathrm{~K}^{p}}\right\}_{\mathrm{K}^{p}}$.

Proof. Vasiu ([27], [28, 29]) and Kisin [14] constructed an integral canonical model $\mathscr{S}_{\mathrm{K}}=\mathscr{S}_{\mathrm{K}}(G, \mathfrak{H})$ of $\mathrm{Sh}_{\mathrm{K}}$ by normalizing $\mathrm{Sh}_{\mathrm{K}}$ in a certain integral model of $\mathrm{Sh}_{\mathrm{K}^{\prime}}\left(\mathrm{GSp}(V, \psi), \mathcal{S}^{ \pm}\right)$(for a suitable choice of $\mathrm{K}^{\prime} \subset \mathrm{GSp}(V, \psi)\left(\mathbb{A}_{\mathrm{f}}\right)$ ) constructed from a Mumford moduli scheme. (The main content is to verify that this construction yields an integral canonical model.) The existence of $\mathcal{A}_{\mathrm{K}_{p} \mathrm{~K}^{p}} \rightarrow$ $\mathscr{S}_{\mathrm{K}_{p} \mathrm{~K}^{p}}$ follows from the construction. For (2), see [14, Corollary 2.3.9]

REMARK 3.3.4. The discussion on Hecke action in Section 3.2.4 can be extended to the prime-to- $p$ Hecke action (that is, the $G\left(\mathbb{A}_{\mathrm{f}}^{p}\right)$-action) on the integral canonical models, which we explain now. Let $\mathrm{K}:=\mathrm{K}_{p} \mathrm{~K}^{p}$ be as before, and pick a geometric point $x$ of $\mathrm{Sh}_{\mathrm{K}}$, viewed also as a geometric point of $\mathscr{S}_{\mathrm{K}}$. As $\mathscr{S}_{\mathrm{K}}$ is normal, the open immersion $\mathrm{Sh}_{\mathrm{K}} \hookrightarrow \mathscr{S}_{\mathrm{K}}$ induces a (surjective) quotient morphism $\pi_{1}^{\text {ét }}\left(\mathrm{Sh}_{\mathrm{K}}, x\right) \rightarrow \pi_{1}^{\text {ét }}\left(\mathscr{S}_{\mathrm{K}}, x\right)$.

Note that the lisse sheaf $\mathcal{V}_{\mathbb{A}_{\mathrm{f}}^{p}}\left(\mathcal{A}_{\mathrm{K}, E}\right)$ on $\mathrm{Sh}_{\mathrm{K}}$ extends to a lisse sheaf $\mathcal{V}_{\mathbb{A}_{\mathrm{f}}^{p}}\left(\mathcal{A}_{\mathrm{K}}\right)$ on $\mathscr{S}_{\mathrm{K}}$. By considering the monodromy action at geometric points, it now follows that the prime-to- $p$ étale tensors $t_{\alpha, \text { êt }}^{\text {univ }}: \mathbf{1} \rightarrow \mathcal{V}_{\mathbb{A}_{\mathrm{f}}^{p}}\left(\mathcal{A}_{\mathrm{K}, E}\right)^{\otimes}$ on the generic fibre extend to the integral canonical model:

$$
t_{\alpha, \text { ét }}^{\text {univ } p}: \mathbf{1} \rightarrow \mathcal{V}_{\mathbb{A}_{\mathrm{f}}^{p}}\left(\mathcal{A}_{\mathrm{K}}\right)^{\otimes} \text {. }
$$

Furthermore, the prime-to- $p$ part of the level structure $\eta_{K^{p}}$ (that is, the image of $\eta_{K}$ in isom $\left(V_{\mathbb{A}_{\mathrm{f}}^{p}}, \mathcal{V}_{\mathbb{A}_{\mathrm{f}}^{p}}\left(\mathcal{A}_{\mathrm{K}, E}\right)\right)$ extends to the integral canonical model:

$$
\eta_{\mathrm{K}^{p}} \in \Gamma\left(\mathscr{S}_{\mathrm{K}}, \operatorname{isom}\left[\left(V_{\mathbb{A}_{\mathrm{f}}^{p}},\left(s_{\alpha}\right)\right),\left(\mathcal{V}_{\mathbb{A}_{\mathrm{f}}^{p}}\left(\mathcal{A}_{\mathrm{K}}\right),\left(t_{\alpha, \mathrm{e} t}^{\text {univ } p}\right)\right)\right] / \mathrm{K}^{p}\right),
$$

which only depends on the prime-to- $p$ isogeny class of $\left(\mathcal{A}_{\mathrm{K}},\left(t_{\alpha \text {,ét }}^{\text {univ, } p}\right)\right)$. Now for any $g \in G\left(\mathbb{A}_{\mathrm{f}}^{p}\right)$, Theorem 3.3 .3 gives an isomorphism $[g]: \mathscr{S}_{g \mathrm{~K} g^{-1}} \stackrel{\sim}{\rightarrow} \mathscr{S}_{\mathrm{K}}$ extending the map on the generic fibre described in Section 3.2.4. Then, we have 
$[g]^{*}\left(\mathcal{A}_{\mathrm{K}},\left(t_{\alpha, \text { ét }}^{\text {uni, }}\right)\right)=\left(\mathcal{A}_{g \mathrm{~K} g^{-1}},\left(t_{\alpha, \text { ét }}^{\text {univ } p}\right)\right)$ as prime-to- $p$ isogeny classes, and the pullback $[g]^{*} \eta_{K^{p}}$ over the integral canonical model has exactly the same description as in Section 3.2.4.

Let us now move on to the $p$-adic part of the (co)homology of $\mathcal{A}_{\mathrm{K}}$ and tensors thereof. Since the $p$-adic lisse sheaf $\mathcal{V}_{\mathbb{Q}_{p}}\left(\mathcal{A}_{\mathrm{K}, E}\right)$ on $\mathrm{Sh}_{\mathrm{K}}$ does not extend over $\mathscr{S}_{\mathrm{K}}$, we cannot extend the $p$-adic étale tensor $t_{\alpha, \text { ét }, p}^{\text {univ }}: \mathbf{1} \rightarrow \mathcal{V}_{\mathbb{Q}_{p}}\left(\mathcal{A}_{\mathrm{K}, E}\right)^{\otimes}$ to the integral canonical model. Instead, one would expect that $t_{\alpha, \text { ét, } p}^{\text {univ }}$ should give rise to a natural crystalline Tate tensor by $p$-adic Hodge theory.

Let $\widehat{\mathscr{S}_{\mathrm{K}}}$ denote the $p$-adic completion of $\mathscr{S}_{\mathrm{K}}$ (that is, the formal completion of $\mathscr{S}_{\mathrm{K}}$ at the special fibre), and $\widehat{f}: \widehat{\mathcal{A}}_{\mathrm{K}} \rightarrow \widehat{\mathscr{S}}_{\mathrm{K}}$ the $p$-adic completion of $f: \mathcal{A}_{\mathrm{K}} \rightarrow$ $\mathscr{S}_{\mathrm{K}}$. Then we have a natural isomorphism $\mathrm{H}_{\mathrm{dR}}^{1}\left(\widehat{\mathcal{A}}_{\mathrm{K}} / \widehat{\mathscr{S}_{\mathrm{K}}}\right) \cong\left(R^{1} \widehat{f}_{\mathrm{CRIS}, *} \mathcal{O}_{\widehat{\mathcal{A}}_{\mathrm{K}} / \mathbb{Z}_{p}}\right) \widehat{\mathscr{S}}_{\mathrm{K}}$, where the right hand side is the pull-back of the crystal to the Zariski site. In particular, the de Rham tensor $\left(t_{\alpha, \mathrm{dR}}^{\text {univ }}\right)$ on $\mathcal{A}_{\mathrm{K}}$ induce an $\mathcal{O}_{\widehat{\mathscr{S}_{\mathrm{K}}} / \mathbb{Z}_{p}}$-linear morphisms of crystals:

$$
t_{\alpha}^{\text {univ }}: \mathbf{1} \rightarrow\left(R^{1} \widehat{f}_{\mathrm{CRIS}, *} \mathcal{O}_{\widehat{\mathcal{A}}_{\mathrm{K}} / \mathbb{Z}_{p}}\right)^{\otimes}=\mathbb{D}\left(\widehat{\mathcal{A}}_{\mathrm{K}}\left[p^{\infty}\right]\right)^{\otimes} .
$$

By construction, $t_{\alpha, \mathrm{dR}}^{\text {univ }}$ coincides with the de Rham tensor associated to $t_{\alpha}^{\text {univ }}$ by Definition 2.7.1.

Proposition 3.3.7. The morphisms $\left(t_{\alpha}^{\mathrm{univ}}\right)$ in (3.3.6) are crystalline Tate tensors on $\widehat{\mathcal{A}}_{\mathrm{K}}$ in the sense of Definition 2.7.2. Furthermore, the p-adic comparison isomorphism matches $\left(t_{\alpha}^{\text {univ }}\right)$ with $\left(t_{\alpha, \mathrm{ét}, p}^{\text {univ }}\right)$.

Proof. This is essentially a corollary of relative crystalline comparison for $p$-divisible groups and the theorem of Blasius and Wintenberger; cf. [2, Theorem 0.3].

Consider the $p$-adic étale tensor

$$
t_{\alpha, \text { ét }, p}^{\text {univ }}: \mathbf{1} \rightarrow \mathcal{V}_{\mathbb{Q}_{p}}\left(\mathcal{A}_{\mathrm{K}, E}\right)^{\otimes} .
$$

Then the relative comparison isomorphism provides an $F$-equivariant morphism of isocrystals

$$
t_{\alpha}: \mathbf{1} \rightarrow\left(\mathbb{D}\left(\mathcal{A}_{\mathrm{K}}\left[p^{\infty}\right]\right)^{*}\right)^{\otimes}[1 / p]=\mathbb{D}\left(\mathcal{A}_{\mathrm{K}}\left[p^{\infty}\right]\right)^{\otimes}[1 / p] .
$$

Indeed, the argument [8, Section 6] can be generalized to prove this; see [11, Theorem 5.3] for the precise statement, which globalizes to show the claim.

It remains to show that $t_{\alpha}=t_{\alpha}^{\text {univ }}$, which can be extracted from the construction of $\mathscr{S}_{\mathrm{K}}$ (cf. the proof of Proposition 2.3.5 and Corollary 2.3.9 in [14]). One can also extract a direct argument from [14] as follows. By smoothness of $\mathscr{S}_{\mathrm{K}}$, both $t_{\alpha}$ 
and $t_{\alpha}^{\text {univ }}$ are determined by the induced sections on $\mathrm{H}_{\mathrm{dR}}^{1}\left(\mathcal{A}_{\mathrm{K}} / \widehat{\mathscr{S}}_{\mathrm{K}}^{\text {rig }}\right)^{\otimes}$, so the claim $t_{\alpha}=t_{\alpha}^{\text {univ }}$ can be verified on the fibres at a Zariski dense set of points of $\widehat{\mathscr{S}}_{\mathrm{K}}^{\text {rig }}$. Indeed, the (classical) points of $\widehat{\mathscr{S}_{\mathrm{K}}^{\text {rig }}}$ which come from $\overline{\mathbb{Q}}$-points of $\mathscr{S}_{\mathrm{K}}$ is Zariski dense in $\widehat{\mathscr{S}_{\mathrm{K}}^{\text {irg }}}$, and the fibres of $t_{\alpha}$ and $t_{\alpha}^{\text {univ }}$ (at $\overline{\mathbb{Q}}$-points) coincide by the theorem of Blasius and Wintenberger [2, Theorem 0.3].

We fix an embedding $\kappa(\mathfrak{p}) \hookrightarrow \overline{\mathbb{F}}_{p}$, and set $W:=W\left(\overline{\mathbb{F}}_{p}\right)$ and $K_{0}=$ Frac $W$. Let $\sigma$ denote the Witt vectors Frobenius endomorphism on $W$ and $K_{0}$.

For $\mathrm{K}=\mathrm{K}_{p} \mathrm{~K}^{p}$ with $\mathrm{K}_{p}=G\left(\mathbb{Z}_{p}\right)$, we consider $\tilde{x}$ : Spec $W \rightarrow \mathscr{S}_{\mathrm{K}}$, and let $x$ denote the $\overline{\mathbb{F}}_{p}$-point induced by $\tilde{x}$. Let $\mathcal{A}_{\mathrm{K}, \tilde{x}}$ and $\mathcal{A}_{\mathrm{K}, x}$ respectively denote the pullback of $\mathcal{A}_{\mathrm{K}}$.

The following result was originally conjectured by Milne and was proved by Vasiu and Kisin (independently) in the course of constructing $\mathscr{S}_{\mathrm{K}}$ (that is, proving Theorem 3.3.3):

Proposition 3.3.8. There is a $W$-linear isomorphism

$$
W \otimes_{\mathbb{Z}_{(p)}} \Lambda_{\mathbb{Z}_{(p)}}^{*} \cong \mathbb{D}\left(\mathcal{A}_{\mathrm{K}, \tilde{x}}\left[p^{\infty}\right]\right)(W)
$$

matching $\left(1 \otimes s_{\alpha}\right)$ and $\left(t_{\alpha, \mathrm{dR}, \tilde{x}}^{\mathrm{univ}}\right)$. In particular, the pointwise stabilizer of $\left(t_{\alpha, \mathrm{dR}, \tilde{x}}^{\mathrm{univ}}\right)$ in $\operatorname{GL}\left(\mathbb{D}\left(\mathcal{A}_{\mathrm{K}, x}\left[p^{\infty}\right]\right)(W)\right.$ is isomorphic to $G_{W}$.

Proof. We first show that there exists an isomorphism

$$
\mathbb{Z}_{p} \otimes_{\mathbb{Z}_{(p)}} \Lambda_{\mathbb{Z}_{(p)}} \cong T_{p}\left(\mathcal{A}_{\mathrm{K}, \tilde{x}}\right)
$$

which matches $\left(1 \otimes s_{\alpha}\right)$ and $\left(t_{\alpha, \text { ét, } p}^{\text {univ }}\right)$. Indeed, by fixing an embedding $\tau: W \hookrightarrow \mathbb{C}$ we obtain an isomorphism

$$
T_{p}\left(\mathcal{A}_{\mathrm{K}, \tilde{x}}\right) \cong \mathrm{H}_{1}\left(\tau^{*} \mathcal{A}_{\mathrm{K}, \tilde{x}}, \mathbb{Z}_{p}\right)
$$

matching $\left(t_{\alpha, \text { êt }, p}^{\text {univ }}\right)$ and the 'Betti tensors' $\left(t_{\alpha, B}^{\text {univ }}\right)$ constructed in Lemma 3.1.5. Now by construction, there exists an isomorphism $\mathbb{Z}_{p} \otimes_{\mathbb{Z}_{(p)}} \Lambda_{\mathbb{Z}_{(p)}} \cong \mathrm{H}_{1}\left(\tau^{*} \mathcal{A}_{\mathrm{K}, \tilde{x}}, \mathbb{Z}_{p}\right)$ matching the tensors.

Now it remains to show the existence of an isomorphism

$$
W \otimes_{\mathbb{Z}_{p}} T_{p}\left(\mathcal{A}_{\mathrm{K}, \tilde{x}}\right)^{*} \cong \mathbb{D}\left(\mathcal{A}_{\mathrm{K}, x}\left[p^{\infty}\right]\right)(W)
$$

matching $\left(1 \otimes t_{\alpha, \text { ét, } p}^{\text {univ }}\right)$ and $\left(t_{\alpha}^{\text {univ }}\right)$. Since these étale and crystalline tensors are related by the $p$-adic comparison isomorphism by Proposition 3.3.7, the existence of such an isomorphism was proved by Vasiu and Kisin in the course of constructing integral canonical models; cf. [14, Proposition 1.3.4], [30]. 
We now extend the $G$-torsor $P_{\mathrm{K}, E}$ over $\mathrm{Sh}_{\mathrm{K}}$ (Section 3.2.1) to the integral canonical model $\mathscr{S}_{\mathrm{K}}$. Consider the following closed subscheme of the isom scheme over $\mathscr{S}_{\mathrm{K}}$

$$
P_{\mathrm{K}} \subset \operatorname{isom}_{\mathscr{S}_{\mathrm{K}}}\left(\mathcal{V}_{\mathrm{dR}}\left(\mathcal{A}_{\mathrm{K}}\right), \mathcal{O}_{\mathscr{S}_{\mathrm{K}}} \otimes_{\mathbb{Z}_{(p)}} \Lambda_{\mathbb{Z}_{(p)}}\right),
$$

defined by the condition of matching $\left(t_{\alpha, \mathrm{dR}}^{\text {univ }}\right) \subset \Gamma\left(\mathscr{S}_{\mathrm{K}}, \mathcal{V}_{\mathrm{dR}}\left(\mathcal{A}_{\mathrm{K}}\right)^{\otimes}\right)$ with $\left(s_{\alpha}\right) \subset \Lambda_{\mathbb{Z}_{(p)}}^{\otimes}$ for each $\alpha$. Then we have $P_{\mathrm{K}, E}=P_{\mathrm{K}} \times_{\operatorname{Spec} \mathscr{O}_{E,(\mathfrak{p})}} \operatorname{Spec} E$, which is a $G$-torsor over $\mathrm{Sh}_{\mathrm{K}}$.

Lemma 3.3.10. The scheme $P_{\mathrm{K}}$ above is a G-torsor over $\mathscr{S}_{\mathrm{K}}$.

Proof. It follows from the construction of $\mathscr{S}_{\mathrm{K}}$ (cf. [15, Proposition 1.3.9(1)]) and Proposition 3.3.8 that $P_{\mathrm{K}}$ pulls back to a $G$-torsor over the completion of $\mathscr{S}_{\mathrm{K}}$ at any $\overline{\mathbb{F}}_{p}$-point. This proves the claim.

Next, we need a notion of $\{\mu\}$-filtrations over $\mathscr{S}_{\mathrm{K}}$. Recall that the conjugacy class $\{\mu\}$ of $\mu_{h}$ for some $h \in \mathfrak{H}$ (by the recipe given in (3.1.4)) is independent of the choice of $h$ and defined over $E$. Therefore, by choosing a place $\mathfrak{p} \mid p$ of $E$ we may view $\{\mu\}$ as a conjugacy class of cocharacters over $\bar{E}_{\mathfrak{p}}$. Clearly, this conjugacy class $\{\mu\}$ is defined over $E_{\mathfrak{p}}$.

We obtain the following lemma using the assumption that $G$ is unramified at $p$.

Lemma 3.3.11. The geometric conjugacy class $\{\mu\}$ over $\bar{E}_{\mathfrak{p}}$ contains a cocharacter $\mu: \mathbb{G}_{m} \rightarrow G_{\mathscr{O}_{E_{\mathfrak{p}}}}$ defined over the valuation ring $\mathscr{O}_{E_{\mathfrak{p}}}$.

Proof. By [17, Lemma 1.1.3(a)], the geometric conjugacy class $\{\mu\}$ contains a cocharacter $\mu^{\prime}: \mathbb{G}_{m} \rightarrow G_{E_{\mathfrak{p}}}$ defined over $E_{\mathfrak{p}}$ (since $\{\mu\}$ is defined over $E_{\mathfrak{p}}$ and $G_{\mathbb{Q}_{p}}$ is quasisplit by unramifiedness). Let $S \subset G_{E_{\mathfrak{p}}}$ be a maximal $E_{\mathfrak{p}}$-split torus containing the image of $\mu^{\prime}$. Since any maximal $E_{\mathfrak{p}}$-split tori are $G\left(E_{\mathfrak{p}}\right)$-conjugate to each other (cf. [3, Theorem 20.9(ii)]), there exists $g \in G\left(E_{\mathfrak{p}}\right)$ such that ${ }^{g} S:=$ $g S g^{-1}$ is the generic fibre of a maximal split torus in $G_{\mathscr{O}_{E \mathfrak{p}}}$. We set $\mu:={ }^{g} \mu^{\prime}$, which extends over $\mathscr{O}_{E_{\mathfrak{p}}}$.

Corollary 3.3.12. The Hodge filtration for $\mathcal{A}_{\mathrm{K}} \rightarrow \mathscr{S}_{\mathrm{K}}$ is a $\{\mu\}$-filtration with respect to $\left(t_{\alpha, \mathrm{dR}}\right)$, where $\{\mu\}$ is the $G(W)$-conjugacy class of $\mu$ as in Lemma 3.3.11.

Proof. By Lemmas 3.3.10 and 3.3.11, $\{\mu\}$-filtrations in $\mathcal{V}_{\mathrm{dR}}\left(\mathcal{A}_{\mathrm{K}}\right)^{*}$ form a smooth closed subscheme of a certain grassmannian over $\mathscr{S}_{\mathrm{K}}$ (cf. Lemma 2.5.6). Since the Hodge filtration for $\mathcal{A}_{\mathrm{K}, E} \rightarrow \mathrm{Sh}_{\mathrm{K}}$ is a $\{\mu\}$-filtration (cf. Section 3.2.2), to prove the corollary it suffices to show that the Hodge filtration of $\mathcal{A}_{\mathrm{K}}$ becomes a $\{\mu\}$-filtration after pulling back by any morphism $\tilde{x}: \operatorname{Spec} R \rightarrow \mathscr{S}_{\mathrm{K}}$, where $R$ is a $p$-adic discrete valuation ring. But this follows from the valuative criterion for properness (applied to the grassmannian of $\{\mu\}$-filtrations over $\mathscr{S}_{\mathrm{K}}$ ). 
3.3.13. We fix $x \in \mathscr{S}_{\mathrm{K}}\left(\overline{\mathbb{F}}_{p}\right)$ and $\tilde{x} \in \mathscr{S}_{\mathrm{K}}(W)$ as before, and write $\mathbb{X}:=\mathcal{A}_{\mathrm{K}, x}\left[p^{\infty}\right]$ and $\widetilde{\mathbb{X}}:=\mathcal{A}_{\mathrm{K}, \tilde{x}}\left[p^{\infty}\right]$. We choose a $W$-isomorphism $\mathbb{D}(\mathbb{X})(W) \cong W \otimes_{\mathbb{Z}_{(p)}} \Lambda_{\mathbb{Z}_{(p)}}^{*}$ matching $\left(t_{\alpha, \tilde{x}}^{\text {univ }}\right)$ and $\left(1 \otimes s_{\alpha}\right)$, as in Proposition 3.3.8. Then we obtain $b \in$ $\operatorname{GL}\left(K_{0} \otimes_{\mathbb{Z}_{(p)}} \Lambda_{\mathbb{Z}_{(p)}}\right)$ such that $F=b(\sigma \otimes \mathrm{id})$. Since each of $\left(t_{\alpha, \tilde{x}}^{\text {univ }}\right)$ is fixed by $F$, it follows that $b$ fixes each of $\left(1 \otimes s_{\alpha}\right)$; that is, we have $b \in G\left(K_{0}\right)$. By Corollary 3.3.12, the Hodge filtration $\mathrm{Fi} \mathrm{I}_{\widetilde{\mathrm{X}}}^{1}$ is induced by ${ }^{g} \mu$ for some $g \in G(W)$ where $\mu$ is a cocharacter as in Lemma 3.3.11.

Lemma 3.3.14. In the above setting, we have $b \in G(W)\left(p^{-1}\right)^{\sigma^{*} \mu} G(W)$.

Proof. This lemma follows from [12, Lemma 2.5.7(2)], which can be applied thanks to Corollary 3.3.12 and Proposition 3.3.8.

\section{Rapoport-Zink uniformization via formal schemes}

In this section, we relate Rapoport-Zink spaces of Hodge type constructed in [12] with a certain completion of $\mathscr{S}_{\mathrm{K}}$ (cf. Theorem 4.7), generalizing (the unramified case of) Rapoport-Zink uniformization of PEL Shimura varieties (cf. [26, Theorem 6.23]).

Using Kisin's theorem on quasi-isogeny groups of abelian varieties with tensors (which we recall in Theorem 4.8), we refine the uniformization; namely, we descend the uniformization over $\mathscr{O}_{E, \mathfrak{p}}$ (Section 4.9). (See [26, Proposition 6.16] for the PEL case.)

From now on, we always assume that $p>2$ without mentioning it.

4.1. Review of Rapoport-Zink spaces of Hodge type. We recall the definitions and main results in [12]. We work in the setting of Section 3.3.13.

Let Nilp $_{W}$ be the category of $W$-algebras where $p$ is nilpotent. For $b \in G\left(K_{0}\right)$ and $X_{0}$ as in Section 3.3.13, we define a functor $\mathrm{RZ}_{b}: \mathrm{Nilp}_{W} \rightarrow$ (Sets) so that $\mathrm{RZ}_{b}(R)$ is the set of isomorphism classes of pairs $(X, \iota)$ where $X$ is a $p$-divisible group over $R$ and $\iota: \mathbb{X}_{R / p} \rightarrow X_{R / p}$ is a quasi-isogeny (that is, an invertible global section of $\left.\operatorname{Hom}\left(\mathbb{X}_{R / p}, X_{R / p}\right)\left[\frac{1}{p}\right]\right)$. Note that $\mathrm{RZ}_{b}$ only depends, up to isomorphism, on the $\sigma$-conjugacy class of $b$ in $\operatorname{GL}\left(K_{0} \otimes \Lambda_{\mathbb{Z}_{(p)}}\right)$. By [26, Theorem 2.16], RZ $Z_{b}$ is representable by a formal scheme which is locally formally of finite type and formally smooth over $W$. We also let $\mathrm{R} \mathrm{Z}_{b}$ denote the representing formal schemes.

REMARK 4.1.1. For any $p$-divisible group $X$ over $R \in \mathfrak{A} \mathfrak{R}_{W}$ which lifts $\mathbb{X}$, there exists a unique quasi-isogeny $\mathbb{X}_{R / p} \rightarrow X_{R / p}$ lifting the identification $\mathbb{X} \stackrel{\sim}{\rightarrow} X_{\overline{\mathbb{F}}_{p}}$. This identifies the universal deformation space of $\mathbb{X}$ with the completion of $\mathrm{RZ}_{b}$ at the point $(\mathbb{X}$, id $) \in \mathrm{RZ}_{b}\left(\overline{\mathbb{F}}_{p}\right)$; cf. [26, Proposition 3.33]. 
Let $s_{\alpha, \mathbb{D}}\left(:=t_{\alpha, x}^{\text {univ }}\right): \mathbf{1} \rightarrow \mathbb{D}(\mathbb{X})^{\otimes}$ be the crystalline Tate tensors induced from $\left(t_{\alpha}^{\text {univ }}\right)$ on $\widehat{\mathcal{A}}_{\mathrm{K}}$; that is, we have $s_{\alpha, \mathbb{D}}(W)=1 \otimes s_{\alpha}$ under the identification as in Proposition 3.3.8, where $\left(s_{\alpha}\right) \subset \Lambda^{\otimes}$ define $G$. Then, for any $(X, \iota) \in \mathrm{RZ}_{b}(R)$ with $R \in$ Nilp $_{W}$, we have a Frobenius-equivariant morphism of isocrystals $s_{\alpha, \mathbb{D}}: \mathbf{1} \rightarrow$ $\mathbb{D}(X)^{\otimes}\left[\frac{1}{p}\right]$ which uniquely lifts

$$
s_{\alpha, \mathbb{D}}: \mathbf{1} \stackrel{\left(s_{\alpha, \mathbb{D}}\right)_{R / p}}{\longrightarrow} \mathbb{D}\left(X_{0, R / p}\right)^{\otimes}\left[\frac{1}{p}\right] \stackrel{\mathbb{D}(t)^{-1}}{\longrightarrow} \mathbb{D}\left(X_{R / p}\right)^{\otimes}\left[\frac{1}{p}\right]
$$

alternatively, one may uniquely lift $\iota$ to $\tilde{\iota}: \widetilde{\mathbb{X}}_{R} \rightarrow X$ and obtain $s_{\alpha, \mathbb{D}}$ from the tensor on $\widetilde{\mathbb{X}}$.

In general, there may not exist any morphism of (integral) crystals giving rise to $s_{\alpha, \mathbb{D}}$. On the other hand, there is a natural closed formal subscheme of $\mathrm{RZ}_{b}$ over which $\left(s_{\alpha, \mathbb{D}}\right)$ is induced from crystalline Tate tensors.

Theorem 4.1.3 [12, Theorem 4.9.1]. Assume that $p>2$. Then there exists a closed formal subscheme $\mathrm{RZ}_{G, b} \subset \mathrm{RZ}_{b}$, which is formally smooth over $W$, with the following universal property: Let $R$ be a formally smooth formally finitely generated algebra over either $W$ or $W / p^{m}$, and consider a morphism $f: \operatorname{Spf} R \rightarrow \mathrm{RZ}_{b}$. Let $X$ be a p-divisible group over $\operatorname{Spec} R$ which pulls back to $f^{*} X_{\mathrm{Rz}_{b}}$ over $\operatorname{Spf} R$. Then $f$ factors through $\mathrm{RZ}_{G, b}$ if and only if there exists a crystalline Tate tensors $t_{\alpha}: \mathbf{1} \rightarrow \mathbb{D}(X)^{\otimes}$ for each $\alpha$ such that

(1) For some ideal of definition $J$ of $R$ containing $p$, the pull-back of $t_{\alpha}$ over $R / J$ induces the map of isocrystals $s_{\alpha, \mathbb{D}}: \mathbf{1} \rightarrow \mathbb{D}\left(X_{R / J}\right)^{\otimes}\left[\frac{1}{p}\right]$.

(2) Let $\widetilde{R}$ be a p-adic lift of $R$ which is formally smooth over $W$. Then the $\widetilde{R}$-scheme

$$
P_{\widetilde{R}}:=\operatorname{isom}_{\widetilde{R}}\left[\left(\mathbb{D}(X)(\widetilde{R}),\left(t_{\alpha}(\widetilde{R})\right)\right],\left[\widetilde{R} \otimes_{\mathbb{Z}_{p}} \Lambda^{*},\left(1 \otimes s_{\alpha}\right)\right]\right),
$$

defined as in (2.5.4), is a G-torsor.

(3) The Hodge filtration $\mathrm{Fil}_{X}^{1} \subset \mathbb{D}(X)(R)$ is a $\{\mu\}$-filtration with respect to $\left(t_{\alpha, \mathrm{dR}}\right) \subset \mathbb{D}(X)(R)^{\otimes}$.

The closed formal subscheme $\mathrm{RZ}_{G, b} \subset \mathrm{RZ}$ is independent of the choice of $\left(s_{\alpha}\right)$.

4.1.4. Let $f: \operatorname{Spf} R \rightarrow \mathrm{RZ}_{G, b}$ be as in Theorem 4.1.3 and we use the notation as above. Then $\left(t_{\alpha}\right)$ are uniquely determined by $f$ (cf. [12, Lemma 4.6.4]). Therefore, applying the universal property to an open affine covering of $\mathrm{RZ}_{G, b}$ we obtain a 'universal family' of crystalline Tate tensors

$$
t_{\alpha}: \mathbf{1} \rightarrow \mathbb{D}\left(\left.\left(X_{\mathrm{Rz}_{b}}\right)\right|_{\mathrm{Rz}_{G, b}}\right)^{\otimes} .
$$


Let us recall the description of $\mathrm{RZ}_{G, b}\left(\overline{\mathbb{F}}_{p}\right)$ and the formal completion $\widehat{\mathrm{RZ}}_{G, b, y}$ at $y \in \mathrm{RZ}_{G, b}\left(\overline{\mathbb{F}}_{p}\right)$ from $[12$, Section 4.8].

If we fix an isomorphism $\left[\mathbb{D}(\mathbb{X})(W),\left(s_{\alpha, \mathbb{D}}\right)\right] \cong\left[W \otimes_{\mathbb{Z}_{p}} \Lambda^{*},\left(1 \otimes s_{\alpha}\right)\right]$, then $(X, \iota) \in \mathrm{RZ}_{G, b}\left(\overline{\mathbb{F}}_{p}\right)$, the quasi-isogeny $\iota$ induces a map $g \in G\left(K_{0}\right)$ on $W \otimes_{\mathbb{Z}_{p}} \Lambda^{*}$. Since the choice of the isomorphism $\left[\mathbb{D}(\mathbb{X})(W),\left(s_{\alpha, \mathbb{D}}\right)\right] \cong\left[W \otimes_{\mathbb{Z}_{p}} \Lambda^{*},\left(1 \otimes s_{\alpha}\right)\right]$ admits a simply transitive $G(W)$-action, we get an injective map $\mathrm{RZ}_{G, b}\left(\overline{\mathbb{F}}_{p}\right) \hookrightarrow$ $G\left(K_{0}\right) / G(W)$. And its image is given by the following affine Deligne-Lusztig set (cf. [12, Proposition 2.5.9]):

$$
\mathrm{RZ}_{G, b}\left(\overline{\mathbb{F}}_{p}\right) \stackrel{\sim}{\rightarrow}\left\{g \in G\left(K_{0}\right) \mid g^{-1} b \sigma(g) \in G(W) b G(W)\right\} / G(W) .
$$

Now, given $y \in \mathrm{RZ}_{G, b}\left(\overline{\mathbb{F}}_{p}\right)$, one can identify the formal completion $\widehat{\mathrm{RZ}}_{G, b, y}$ with the explicit deformation space with Tate tensors constructed by Faltings; cf. [12, Section 4.8]. Instead of recalling the precise description, let us record the following consequence which will be used later.

Let $\widehat{\mathscr{S}}_{\mathrm{K}, x}$ denote the completion of $\mathscr{S}_{\mathrm{K}}$ at $x: \operatorname{Spec} \overline{\mathbb{F}}_{p} \rightarrow \mathscr{S}_{\mathrm{K}}$. We also view $x=$ $(\mathbb{X}$, id $) \in \mathrm{RZ}_{G, b}\left(\overline{\mathbb{F}}_{p}\right)$. As observed in Remark 4.1.1, we have a morphism $\widehat{\mathscr{S}}_{\mathrm{K}, x} \rightarrow$ $\widehat{\mathrm{RZ}}_{b, x}$ given by rigidity of quasi-isogeny, which is a closed immersion of formal schemes by Serre-Tate deformation theory [10, Theorem 1.2.1]. Furthermore, this closed immersion factors through $\widehat{\mathrm{RZ}}_{G, b, x}$ by the universal property of $\mathrm{RZ}_{G, b}$ (Theorem 4.1.3). Indeed, the crystalline Tate tensors $\left(t_{\alpha}^{\text {univ }}\right)$ on $\widehat{\mathcal{A}}_{\mathrm{K}}$ induce the required $\left(t_{\alpha}\right)$, which satisfy (1) by taking $J$ to be the maximal ideal and the remaining conditions by Corollary 3.3.12.

Proposition 4.1.6. The morphism of formal schemes $\widehat{\mathscr{S}}_{\mathrm{K}, x} \rightarrow \widehat{\mathrm{RZ}}_{G, b, x}$, defined above, is an isomorphism.

Proof. Note that both completions as well as the deformations of $\mathbb{X}$ over them have the same explicit description, and the morphism we constructed match them; cf. [12, Theorem 4.9.1] and [15, Proposition 1.3.9(1)].

4.1.7. Assume that there is another embedding $(G, \mathfrak{H}) \hookrightarrow\left(\mathrm{GSp}\left(V^{\prime}, \psi^{\prime}\right), \mathcal{S}^{\prime \pm}\right)$ of Shimura data and a $\mathbb{Z}_{(p)}$-lattice $\Lambda_{\mathbb{Z}_{(p)}^{\prime}}^{\prime} \subset V^{\prime}$ as in Section 3.3.1. This choice gives rise to another abelian scheme $\mathcal{A}_{\mathrm{K}}^{\prime}$ over $\mathscr{S}_{\mathrm{K}}$. We set $\mathbb{X}^{\prime}:=\mathcal{A}_{\mathrm{K}, x}^{\prime}$ for $x \in \mathscr{S}_{\mathrm{K}}\left(\overline{\mathbb{F}}_{p}\right)$ and let $\mathrm{RZ}_{G, b}^{\prime} \subset \mathrm{RZ} \mathrm{Z}_{b}^{\prime}$ denote the moduli spaces constructed using $\mathbb{X}^{\prime}$ instead. Then the functoriality part of [12, Theorem 4.9.1] shows that there exists a unique isomorphism $\mathrm{RZ}_{G, b} \cong \mathrm{RZ}_{G, b}^{\prime}$ respecting the description of $\overline{\mathbb{F}}_{p}$-points and the completions thereof given in (4.1.5) and Proposition 4.1.6.

We recall some of the extra structures that $\mathrm{R} Z_{G, b}$ possesses. 
4.1.8. By classical Dieudonné theory, $J_{b}\left(\mathbb{Q}_{p}\right)$ (2.6.4) can be identified with the group of quasi-isogenies of $\mathbb{X}$ that preserve the crystalline Tate tensors $\left(s_{\alpha, \mathbb{D}}\right)$. It turns out to be a $\mathbb{Q}_{p}$-point of an inner form of a Levi subgroup of $G$; cf. [26, Corollary 1.14]. We define the left action of $J_{b}\left(\mathbb{Q}_{p}\right)$ on $\mathrm{RZ}_{G, b}$ as follows. For any $(X, \iota) \in \mathrm{RZ}_{G, b}(R)$ and $g \in J_{b}\left(\mathbb{Q}_{p}\right)$, we set

$$
g(X, \iota)=\left(X, \iota \circ g^{-1}\right) \text {. }
$$

4.1.9. Put $d:=\left[E_{\mathfrak{p}}: \mathbb{Q}_{p}\right]$, and let $q=p^{d}$ be the cardinality of the residue field of $E_{\mathfrak{p}}$. Let $\tau=\sigma^{d} \in \operatorname{Gal}\left(K_{0} / E_{\mathfrak{p}}\right)$ denote the $q$-Frobenius element (that is, the lift of the $q$ th power map on $\overline{\mathbb{F}}_{p}$ ). For any formal scheme $\mathfrak{X}$ over $\operatorname{Spf} W$, we write $\mathfrak{X}^{\tau}:=\mathfrak{X} \times_{\text {Spf } W, \tau}$ Spf $W$. For any $R \in \operatorname{Nilp}_{W}$, note that $\mathfrak{X}^{\tau}(R)=\mathfrak{X}\left(R^{\tau}\right)$, where $R^{\tau}$ is $R$ viewed as a $W$-algebra via $\tau$. By Weil descent datum over $\mathscr{O}_{E, \mathfrak{p}}$ we mean an isomorphism $\Phi: \mathfrak{X} \rightarrow \mathfrak{X}^{\tau}$. Note that if there exists an $\mathscr{O}_{E, \mathfrak{p}}$-formal scheme $\mathfrak{X}_{0}$ with $\left(\mathfrak{X}_{0}\right)_{W} \cong \mathfrak{X}$, then $\mathfrak{X}$ has a Weil descent datum over $\mathscr{O}_{E, \mathfrak{p}}$. Such a Weil descent datum is called effective.

We define a Weil descent datum $\Phi: \mathrm{RZ}_{b} \stackrel{\sim}{\rightarrow} \mathrm{RZ} Z_{b}^{\tau}$ over $\mathscr{O}_{E, \mathfrak{p}}$, sending $(X, \iota) \in$ $\mathrm{RZ}_{G, b}(R)$ to $\left(X^{\Phi}, \iota^{\Phi}\right) \in \mathrm{RZ}_{G, b}\left(R^{\tau}\right)$, where $X^{\Phi}$ is $X$ viewed as a $p$-divisible group over $R^{\tau}$, and $\iota^{\Phi}$ is the following quasi-isogeny:

$$
\iota_{R^{\tau} / p}^{\Phi}: \mathbb{X}_{R^{\tau} / p}=\left(\tau^{*} \mathbb{X}\right)_{R / p} \stackrel{\text { Frob }^{-d}}{\rightarrow} X_{R / p} \stackrel{\iota}{\rightarrow} X_{R / p}=X_{R / p}^{\Phi},
$$

where Frob $^{d}: \mathbb{X} \rightarrow \tau^{*} \mathbb{X}$ is the relative $q$-Frobenius (with $q=p^{d}$ ). One can check that $\Phi$ restricts to a Weil descent datum $\Phi: \mathrm{RZ}_{G, b} \stackrel{\sim}{\rightarrow} \mathrm{RZ} \mathrm{Z}_{G, b}^{\tau}$ over $\mathscr{O}_{E, \mathfrak{p}}$ (by looking at $\overline{\mathbb{F}}_{p}$-points and the formal completions thereof; cf. [12, Definition 7.3.5]).

Clearly the $J_{b}\left(\mathbb{Q}_{p}\right)$ action commutes with the Weil descent datum $\Phi$. Note that $\Phi$ is not an effective Weil descent datum for $\mathrm{RZ}_{G, b}$.

4.1.10. Over the rigid analytic generic fibre of $R Z_{G, b}$ we can naturally define a tower of étale coverings with Galois group $G\left(\mathbb{Z}_{p}\right)$ equipped with a natural $G\left(\mathbb{Q}_{p}\right)$-action. The $J_{b}\left(\mathbb{Q}_{p}\right)$-action and the Weil descent datum naturally lifts to each layer of the tower in a compatible way. We give a brief review when we use it (Section 5.2), and see [12, Section 7.4] for the details.

4.2. Isogeny classes of $\bmod \boldsymbol{p}$ points. We continue to work in the setting of Section 3.3.13. Let $\iota: \mathcal{A} \rightarrow \rightarrow \mathcal{A}^{\prime}$ be a quasi-isogeny of abelian schemes over $R \in \operatorname{Nilp}_{W}$; that is, an invertible global section of $\operatorname{Hom}\left(\mathcal{A}, \mathcal{A}^{\prime}\right) \otimes_{\mathbb{Z}} \mathbb{Q}$. Then $\iota$ induces the following isomorphisms:

$$
\begin{aligned}
\mathbb{D}\left(\mathcal{A}\left[p^{\infty}\right]\right)[1 / p] & \stackrel{\sim}{\leftarrow} \mathbb{D}\left(\mathcal{A}^{\prime}\left[p^{\infty}\right]\right)[1 / p] ; \\
\mathcal{V}_{\mathbb{A}_{\mathrm{f}}^{p}}(\mathcal{A}) & \stackrel{\sim}{\rightarrow} \mathcal{V}_{\mathbb{A}_{\mathrm{f}}^{p}}\left(\mathcal{A}^{\prime}\right) .
\end{aligned}
$$


Definition 4.2.2. We define an equivalence relations $x \sim x^{\prime}$ for $x, x^{\prime} \in \mathscr{S}_{\mathrm{K}}\left(\overline{\mathbb{F}}_{p}\right)$ if there exists a quasi-isogeny $\iota: \mathcal{A}_{\mathrm{K}, x} \rightarrow-\rightarrow \mathcal{A}_{\mathrm{K}, x^{\prime}}$ such that the isomorphisms (4.2.1) induced by $\iota$ matches $\left(t_{\alpha, x}^{\text {univ }}\right)$ with $\left(t_{\alpha, x^{\prime}}^{\text {univ }}\right)$, and $\left(t_{\alpha, \text { ét }, x}^{\text {univ },}\right)$ with $\left(t_{\alpha, \text { ét, } x^{\prime}}^{\text {univ } p}\right)$. An equivalence class $\phi$ containing $x \in \mathscr{S}_{\mathrm{K}}\left(\overline{\mathbb{F}}_{p}\right)$ is called an isogeny class of $x$.

Let $(X, \iota) \in \mathrm{RZ}_{G, b}(R)$ for $R \in \operatorname{Nilp}_{W}$, and for the choice of the $W$-lift $\widetilde{\mathbb{X}}$ as in Section 3.3.13 let $\tilde{\iota}: \widetilde{\mathbb{X}}_{R} \rightarrow X$ denote the unique lift of $\iota$. Assume that $p^{n} \tilde{\iota}: \widetilde{\mathbb{X}}_{R} \rightarrow$ $X$ is an isogeny, and let

$$
\mathcal{A}:=\left(\mathcal{A}_{\mathrm{K}, \tilde{x}}\right)_{R} / \operatorname{ker}\left(p^{n} \tilde{\imath}\right)
$$

be an abelian scheme over $R$. Note that $\mathcal{A}\left[p^{\infty}\right]=X$ by construction, and we have a quasi-isogeny

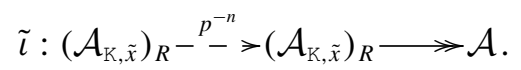

Note that $\tilde{\iota}$ induces crystalline Tate tensors $t_{\alpha}: \mathbf{1} \rightarrow \mathbb{D}\left(\mathcal{A}\left[p^{\infty}\right]\right)^{\otimes}\left[\frac{1}{p}\right]$, and an isomorphism of $\mathbb{A}_{\mathrm{f}}^{p}$-local systems

$$
\mathcal{V}_{\mathbb{A}_{\mathrm{f}}^{p}}(\tilde{\iota}): \mathcal{V}_{\mathbb{A}_{\mathrm{f}}^{p}}\left(\left(\mathcal{A}_{\mathrm{K}, \tilde{x}}\right)_{R}\right) \stackrel{\sim}{\rightarrow} \mathcal{V}_{\mathbb{A}_{\mathrm{f}}^{p}}(\mathcal{A})
$$

Via this isomorphism, $\left(t_{\alpha, \text { ét }, \tilde{x}}^{\text {univ }}\right)$ induces prime-to- $p$ étale tensors on $\mathcal{A}$ as follows

$$
t_{\alpha}^{p}:=\mathcal{V}_{\mathbb{A}_{\mathrm{f}}^{p}}(\tilde{\iota}) \circ t_{\alpha, \text { ét }, \tilde{x}}^{\mathrm{univ}},
$$

and the prime-to- $p$ level structure $\tilde{x}^{*} \eta_{\mathrm{K}^{p}}$ (3.3.5) induces

$$
\eta^{p}=\mathcal{V}(\tilde{\imath}) \circ\left(\tilde{x}^{*} \eta_{\mathrm{K}^{p}}\right) \in \Gamma\left(\operatorname{Spec} R, \operatorname{isom}\left[\left(V_{\mathbb{A}_{\mathrm{f}}^{p}},\left(s_{\alpha}\right)\right),\left(\mathcal{V}_{\mathbb{A}_{\mathrm{f}}^{p}}(\mathcal{A}),\left(t_{\alpha}^{p}\right)\right)\right] / K^{p}\right) .
$$

The next aim is to construct a morphism of formal schemes $\mathrm{RZ}_{G, b} \rightarrow \widehat{\mathscr{S}}_{\mathrm{K}}$ where the target is the $p$-adic completion of $\mathscr{S}_{\mathrm{K}}$.

Proposition 4.3. There exists a unique morphism of formal schemes

$$
\Theta^{\phi}: \mathrm{RZ}_{G, b} \rightarrow \widehat{\mathscr{S}}_{\mathrm{K}, W}
$$

(depending on $x \in \mathscr{S}_{\mathrm{K}}\left(\overline{\mathbb{F}}_{p}\right)$ but not on the choice of $\Lambda_{\mathbb{Z}_{(p)}}$ and $\left(s_{\alpha}\right)$ ), such that $\Theta^{\phi}$ maps $(X, \iota) \in \mathrm{RZ}_{G, b}(R)$ (with $\left.R \in \operatorname{Nilp}_{W}\right)$ to an $R$-point $f \in \widehat{\mathscr{S}}_{\mathrm{K}}(R)$ with $f^{*}\left(\mathcal{A}_{\mathrm{K}}\right.$, $\left.\left(t_{\alpha}^{\text {univ }}\right), \eta_{K^{p}}\right) \cong\left(\mathcal{A},\left(t_{\alpha}\right), \eta^{p}\right)$ where the target is defined by (4.2.3). Furthermore, $\Theta^{\phi}$ commutes with the Weil descent data over $\mathscr{O}_{E, \mathfrak{p}}=W(\kappa(\mathfrak{p}))$. 
Proof. The unique existence of $\Theta^{\phi}\left(\overline{\mathbb{F}}_{p}\right): \mathrm{RZ}_{G, b}\left(\overline{\mathbb{F}}_{p}\right) \rightarrow \mathscr{S}_{\mathrm{K}}\left(\overline{\mathbb{F}}_{p}\right)$ (depending on $\left.x \in \mathscr{S}_{\mathrm{K}}\left(\overline{\mathbb{F}}_{p}\right)\right)$, as well as independence of choice, follows from [15, Proposition 1.4.4], which was proved using the main result of [5]. (To obtain the map $\mathrm{RZ}_{G, b}\left(\overline{\mathbb{F}}_{p}\right) \rightarrow \mathscr{S}_{\mathrm{K}}\left(\overline{\mathbb{F}}_{p}\right)$ from [15, Proposition 1.4.4], note that $\mathrm{RZ}_{G, b}\left(\overline{\mathbb{F}}_{p}\right)$ can be identified with a certain affine Deligne-Lusztig set by [12, (4.8.1)].) Considering the description of $\Theta^{\phi}$ on the points valued in artin local rings with residue field $\overline{\mathbb{F}}_{p}$, it follows that $\Theta^{\phi}$ should induce the isomorphism $\widehat{\mathrm{RZ}}_{G, b, y} \stackrel{\sim}{\rightarrow} \widehat{\mathscr{S}}_{\mathrm{K}, \Theta^{\phi}(y)}$ for any $y \in \mathrm{RZ}_{G, b}\left(\overline{\mathbb{F}}_{p}\right)$, given by Proposition 4.1 .6 . Note that this isomorphism is independent of the choice of $\Lambda_{\mathbb{Z}_{(p)}}$ and $\left(s_{\alpha}\right)$. Since $\Theta^{\phi}$ is a map between formal schemes locally formally of finite type over $W$, it is determined by the map it induces on the set of $\overline{\mathbb{F}}_{p}$-points and the completions thereof (cf. [12, Lemma 5.4.2]).

It remains to show the existence of $\Theta^{\phi}$ using some suitable choice of $\Lambda_{\mathbb{Z}_{(p)}}$. Indeed, by Zarhin's trick we may assume that $G_{\mathbb{Z}_{(p)}} \subset \operatorname{GSp}:=\operatorname{GSp}\left(\Lambda_{\mathbb{Z}_{(p)}}, \psi\right)$ where $\psi$ is a perfect alternating form on $\Lambda_{\mathbb{Z}_{(p)}}$. Then we may choose an open compact subgroup $\mathrm{K}^{\prime}=\mathrm{K}_{p}^{\prime} \mathrm{K}^{\prime p} \subset \mathrm{GSp}\left(\mathbb{A}_{\mathrm{f}}\right)$ such that $\mathrm{K}_{p}^{\prime}=\mathrm{GSp}\left(\mathbb{Z}_{p}\right)$ and we have a natural closed immersion $\mathrm{Sh}_{\mathrm{K}} \hookrightarrow \mathrm{Sh}_{\mathrm{K}^{\prime}, E}$ (with the obvious notation); cf. [14, Lemma 2.1.2]. Since $\mathrm{K}_{p}^{\prime}$ is hyperspecial maximal, we have an integral canonical model $\mathscr{S}_{\mathrm{K}^{\prime}}$ of $\mathrm{Sh}_{\mathrm{K}^{\prime}}$, and $\mathscr{S}_{\mathrm{K}}$ is the normalization of the Zariski-closure $\mathscr{S}_{\mathrm{K}}^{-}$of $\mathrm{Sh}_{\mathrm{K}}$ in $\mathscr{S}_{\mathrm{K}^{\prime}}$.

Given $x \in \mathscr{S}_{\mathrm{K}}\left(\overline{\mathbb{F}}_{p}\right)$, we let $x \in \mathscr{S}_{\mathrm{K}^{\prime}}\left(\overline{\mathbb{F}}_{p}\right)$ also denote its image. Then we also obtain $\mathrm{RZ}_{\mathrm{GSp}, b} \subset \mathrm{RZ}_{b}$ by working with GSp instead of $G$, and clearly $\mathrm{RZ}_{G, b}$ is a closed formal subscheme of $\mathrm{RZ}_{\mathrm{GSp}, b}$. Now, the desired map for GSp instead of $G$

$$
\mathrm{RZ}_{\mathrm{GSp}, b} \rightarrow \widehat{\mathscr{S}}_{\mathrm{K}^{\prime}, W}
$$

was already constructed in [26, Theorem 6.21]. We want to show that the restriction $\mathrm{RZ}_{G, b} \rightarrow \widehat{\mathscr{S}}_{\mathrm{K}^{\prime}, W}$ factors through $\widehat{\mathscr{S}}_{\mathrm{K}, W}$. This holds on the level of $\overline{\mathbb{F}}_{p^{-}}$ points and the completions thereof, which follows from [15, Proposition 1.4.4] and the formal-local description of $\mathscr{S}_{\mathrm{K}, W}$ as in [14, Proof of Proposition 2.3.5], respectively. This at least shows that the map $\mathrm{RZ}_{G, b} \rightarrow \widehat{\mathscr{S}}_{\mathrm{K}^{\prime}, W}$ factors through the formal closed subscheme $\widehat{\mathscr{S}}_{\mathrm{K}, W}^{-}$. (Note that given a map $f: S \rightarrow R$ of noetherian rings and an ideal $I \subset S$, we can verify $f(I)=0$ on the completion of $R$ at each maximal ideal.) Now, by formal smoothness $\mathrm{RZ}_{G, b}$ is also normal as a locally noetherian formal scheme. Therefore by choosing suitable irreducible formal open affines $\operatorname{Spf} R$ of $\mathrm{RZ}_{G, b}$ and $\operatorname{Spf} S$ of $\widehat{\mathscr{S}}_{\mathrm{K}, W}^{-}$respectively, the map $\mathrm{RZ}_{G, b} \rightarrow \widehat{\mathscr{S}}_{\mathrm{K}, W}^{-}$is locally given by injective maps of domains $S \rightarrow R$ where $R$ is a normal domain. (To see the injectivity, it suffices to work on the level of completions at each maximal ideals, and this follows from [14, Proof of Proposition 2.3.5].) This shows that $\mathrm{RZ}_{G, b} \rightarrow \widehat{\mathscr{S}}_{\mathrm{K}, W}^{-}$lifts to $\mathrm{RZ}_{G, b} \rightarrow \widehat{\mathscr{S}}_{\mathrm{K}, W}$, as $\mathscr{S}_{\mathrm{K}}$ is the normalization of $\mathscr{S}_{\mathrm{K}}^{-}$. 
To show that $\Theta^{\phi}$ commutes with the Weil descent data, note that it suffices to check this for $\Theta_{\mathbb{F}_{p}}^{\phi}$, in which case the claim is more or less clear from the definition. Cf. the proof of [26, Theorem 6.21].

REMARK 4.3.1. In some sense, the proof of [15, Proposition 1.4.4] essentially proves Proposition 4.3, except that $\mathrm{RZ}_{G, b}$ was not defined in [15] and some ad hoc notion for $\mathrm{RZ}_{G, b}(R)$ was used instead. So Proposition 4.3 can be proved by 'repeating' the proof [15, Proposition 1.4.4] in the following way (taking [5] as the main input). By the argument in [15, Section 1.4.10], the map can be extended to $\mathrm{RZ}{ }_{G, b}^{\circ} \rightarrow \widehat{\mathscr{S}}_{\mathrm{K}, W}$, where $\mathrm{RZ}_{G, b}^{\circ}$ is the connected component containing $x=(\mathbb{X}, \mathrm{id})$. Now, it follows from the main result of [5] that the Hecke action at $p$ transitively permutes the connected components of $\mathrm{RZ}_{G, b}$; cf. [15, Proposition 1.2.22].

Corollary 4.3.2. The map $\Theta^{\phi}: \mathrm{RZ}_{G, b} \rightarrow \widehat{\mathscr{S}}_{\mathrm{K}}$ in Proposition 4.3 extends to

$$
\Theta^{\phi}: \mathrm{RZ}_{G, b} \times G\left(\mathbb{A}_{\mathrm{f}}^{p}\right) / \mathrm{K}^{p} \rightarrow \widehat{\mathscr{S}}, W
$$

so that on points over $R \in \operatorname{Nilp}_{W}$ we have $\left(X, \iota, g \mathrm{~K}^{p}\right) \mapsto\left(\mathcal{A},\left(t_{\alpha}\right), \eta^{p} g\right)$. This morphism commutes with the Weil descent data over $\mathscr{O}_{E, \mathfrak{p}}$.

Definition 4.4. Let $I^{\phi}(\mathbb{Q})$ be the group of quasi-isogenies $\mathcal{A}_{\mathrm{K}, x} \rightarrow-\rightarrow \mathcal{A}_{\mathrm{K}, x}$ which preserve $\left(t_{\alpha, x}^{\text {univ }}\right)$ and $\left(t_{\alpha, \text { ét, }, x}^{\text {univ, }}\right)$ over some finite field. (Note that $\mathcal{A}_{\mathrm{K}, x}$ and the tensors are defined over some finite field as they are obtained from a mod $p$ point of $\mathscr{S}_{\mathrm{K}}$.) Note that $I^{\phi}(\mathbb{Q})$ only depends on $\phi$, not on the individual $x$. We view $I^{\phi}(\mathbb{Q})$ naturally as a subgroup of $J_{b}\left(\mathbb{Q}_{p}\right)$ and $G\left(\mathbb{A}_{\mathrm{f}}^{p}\right)$. We let $I^{\phi}(\mathbb{Q})$ act on $\mathrm{RZ}_{G, b} \times G\left(\mathbb{A}_{\mathrm{f}}^{p}\right) / \mathrm{K}^{p}$ via left translation.

REMARK 4.4.1. In the general Hodge-type (non-PEL) setting, it is a nontrivial theorem of Kisin that $I^{\phi}(\mathbb{Q})$ is the $\mathbb{Q}$-points of an inner form of some Levi subgroup of $G$ with explicit description at each place of $\mathbb{Q}$. (This result can be proved much more easily in the PEL case.) We state this result in Theorem 4.8, and it will be used to prove the stronger statement of RapoportZink uniformization; cf. Section 4.9.

Lemma 4.4.2. The subgroup $I^{\phi}(\mathbb{Q}) \subset J_{b}\left(\mathbb{Q}_{p}\right) \times G\left(\mathbb{A}_{\mathrm{f}}^{p}\right)$ is discrete.

Proof. (Compare with the proof of Theorem 6.23 in [26, p. 289].) Note that $J_{b}\left(\mathbb{Q}_{p}\right)$ has an open compact subgroup consisting of isomorphisms of $\mathbb{X}$; namely, $J_{b}\left(\mathbb{Q}_{p}\right) \cap \mathrm{GL}(W \otimes \Lambda)$. Let $U \subset J_{b}\left(\mathbb{Q}_{p}\right) \times G\left(\mathbb{A}_{\mathrm{f}}^{p}\right)$ be an open subgroup such that the image in $G\left(\mathbb{A}_{\mathrm{f}}^{p}\right)$ stabilizes $\prod_{\ell \neq p} T_{\ell}\left(\mathcal{A}_{\mathrm{K}, x}\right)$ and the image in $J_{b}\left(\mathbb{Q}_{p}\right)$ is contained in the open compact subgroup of isomorphisms. This is always possible to arrange 
by replacing $U$ with an open subgroup of finite index. Then $I^{\phi}(\mathbb{Q}) \cap U$ is a finite group since it is a subgroup of the automorphism group of polarized abelian variety $\left(\mathcal{A}_{\mathrm{K}, x}, \lambda\right)$.

Proposition 4.5. Assume that $\mathrm{K}^{p}$ is 'small enough'. Then the quotient

$$
I^{\phi}(\mathbb{Q}) \backslash R Z_{G, b} \times G\left(\mathbb{A}_{\mathrm{f}}^{p}\right) / \mathrm{K}^{p}
$$

is representable by a formal scheme which is locally formally of finite type and formally smooth over $W$, and the Weil descent datum $\Phi$ of $\mathrm{RZ}_{G, b}$ induces a Weil descent datum on this quotient.

The morphism $\Theta^{\phi}: \mathrm{RZ}_{G, b} \times G\left(\mathbb{A}_{\mathrm{f}}^{p}\right) / \mathrm{K}^{p} \rightarrow \widehat{\mathscr{S}_{\mathrm{K}}}$, defined in Corollary 4.3.2, is invariant under the $I^{\phi}(\mathbb{Q})$-action and the induced morphism of formal schemes

$$
\Theta^{\phi}: I^{\phi}(\mathbb{Q}) \backslash \mathrm{RZ} Z_{G, b} \times G\left(\mathbb{A}_{\mathrm{f}}^{p}\right) / \mathrm{K}^{p} \rightarrow \widehat{\mathscr{S}}_{\mathrm{K}, W}
$$

is a monomorphism of functors on $\mathrm{Nilp}_{W}$.

Proof. Let us first show that the quotient $I^{\phi}(\mathbb{Q}) \backslash \mathrm{RZ}_{G, b} \times G\left(\mathbb{A}_{\mathrm{f}}^{p}\right) / \mathrm{K}^{p}$ is representable by a formal algebraic space. Note that

$$
I^{\phi}(\mathbb{Q}) \backslash \mathrm{R} Z_{G, b} \times G\left(\mathbb{A}_{\mathrm{f}}^{p}\right) / \mathrm{K}^{p}=\coprod_{\Gamma} \Gamma \backslash \mathrm{R} Z_{G, b}
$$

where $\Gamma \subset J_{b}\left(\mathbb{Q}_{p}\right)$ runs over discrete subgroups of the form $I^{\phi}(\mathbb{Q}) \cap g \mathrm{~K}^{p} g^{-1}$ for $g \in G\left(\mathbb{A}_{\mathrm{f}}^{p}\right)$. Such a group $\Gamma$ is separated with respect to the profinite topology and discrete by Lemma 4.4.2. Also $\Gamma$ is torsion-free if $\mathrm{K}^{p}$ is 'small enough' (more precisely, if $\mathrm{K}^{p}$ fixes the $n$-torsion points of $\mathcal{A}_{\mathrm{K}, x}$ for some $n \geqslant 3$; cf. the proof of Theorem 6.23 in [26, pp. 289-290]). Then, the $\Gamma$-action on $\mathrm{RZ}_{G, b}$ has no fixed point since the $\Gamma$-action on $\mathrm{RZ}_{b}$ has no fixed point by [26, Corollary 2.35]. We then show that $\Gamma \backslash R Z_{G, b}$ is representable by a formal algebraic space by repeating the proof of [26, Proposition 2.37]. (Alternatively, one may apply [26, Proposition 2.37] to show that the quotient $\Gamma \backslash \mathrm{RZ}_{b}$ is representable by a formal algebraic space and observe that $\mathrm{RZ}_{G, b}$ is a $\Gamma$-stable closed formal subscheme of $\mathrm{RZ}_{b}$.)

It is clear that $\Theta^{\phi}: \mathrm{RZ}_{G, b} \times G\left(\mathbb{A}_{\mathrm{f}}^{p}\right) / \mathrm{K}^{p} \rightarrow \widehat{\mathscr{S}_{\mathrm{K}, W}}$ is invariant under the $I^{\phi}(\mathbb{Q})$ action. We now show that the induced map of formal algebraic spaces

$$
\Theta^{\phi}: I^{\phi}(\mathbb{Q}) \backslash R Z_{G, b} \times G\left(\mathbb{A}_{\mathrm{f}}^{p}\right) / \mathrm{K}^{p} \rightarrow \widehat{\mathscr{S}}, W
$$

is a monomorphism of functors on Nilp ${ }_{W}$. Indeed, the injectivity on $\overline{\mathbb{F}}_{p}$-points is clear from Proposition 4.3, and $\Theta^{\phi}$ induces an isomorphism on the completions at any $\overline{\mathbb{F}}_{p}$-point (by Proposition 4.1.6). The claim now follows from descent and direct limit consideration. 
Note that any algebraic space which is separated and locally quasifinite over a scheme is a scheme (cf. [20, Théorème (A.2)]). This shows that any closed algebraic subspace of $I^{\phi}(\mathbb{Q}) \backslash R Z_{G, b} \times G\left(\mathbb{A}_{\mathrm{f}}^{p}\right) / \mathrm{K}^{p}$ is a scheme, which shows that $I^{\phi}(\mathbb{Q}) \backslash R Z_{G, b} \times G\left(\mathbb{A}_{\mathrm{f}}^{p}\right) / \mathrm{K}^{p}$ can be represented by a formal scheme. (For example, the image of $\mathrm{RZ}_{G, b}(h)^{m, n} \times G\left(\mathbb{A}_{\mathrm{f}}^{p}\right)$ in $I^{\phi}(\mathbb{Q}) \backslash \mathrm{RZ} Z_{G, b} \times G\left(\mathbb{A}_{\mathrm{f}}^{p}\right) / \mathrm{K}^{p}$ is a scheme for each $(m, n)$, where $\mathrm{RZ}_{G, b}(h)^{m, n}$ is introduced in [12, Section 6.1].)

The assertion on the Weil descent datum follows since $I^{\phi}(\mathbb{Q})$ act on $\mathrm{RZ}_{G, b}$ via $I^{\phi}(\mathbb{Q}) \hookrightarrow J_{b}\left(\mathbb{Q}_{p}\right)$ whose action commutes with the Weil descent datum $\Phi$ of $\mathrm{RZ}_{G, b}$. This concludes the proof.

We finish by identifying $I^{\phi}(\mathbb{Q}) \backslash R Z_{G, b} \times G\left(\mathbb{A}_{\mathrm{f}}^{p}\right) / \mathrm{K}^{p}$ as the completion of $\widehat{\mathscr{S}}_{\mathrm{K}, W}$ at a (possibly infinite) chain of closed subschemes. We first recall the following definition:

DEFINITION 4.6. Let $\mathfrak{X}$ be a formal scheme and $\mathcal{Z}:=\left\{Z_{i}\right\}_{i \in \mathbf{I}}$ where $Z_{i} \subset|\mathfrak{X}|$ is a closed subset such that for each $i \in \mathbf{I}$ there are only finitely many $j \in \mathbf{I}$ with $Z_{i} \cap Z_{j} \neq \emptyset$.

We define the completion $\mathfrak{X}_{/ \mathcal{Z}}$ of $\mathfrak{X}$ along $\mathcal{Z}$ to be the following formal scheme. The underlying topological space is

$$
\left|\mathfrak{X}_{/ \mathcal{Z}}\right|:=\bigcup_{i \in \mathbf{I}} Z_{i}
$$

with the direct limit topology. For each $x \in\left|\mathfrak{X}_{/ \mathcal{Z}}\right|$, we consider the open subset of $\left|\mathfrak{X}_{/ \mathcal{Z}}\right|$ :

$$
\mathcal{Z}(x):=\left(\bigcup_{x \in Z_{i}} Z_{i}\right) \backslash\left(\bigcup_{x \notin Z_{i}} Z_{i}\right),
$$

which is also a locally closed subset of $\mathfrak{X}$. We give a formal scheme structure on $\mathcal{Z}(x)$ as the completion of $\mathfrak{X}$ along $\mathcal{Z}(x)$. The formal scheme $\mathfrak{X}_{/ \mathcal{Z}}$ is obtained by glueing these formal schemes on $\mathcal{Z}(x)$ as we vary $x \in\left|\mathfrak{X}_{/ \mathcal{Z}}\right|$.

Note that if the index set $\mathbf{I}$ is finite (that is, $Z:=\bigcup_{i \in \mathbf{I}} Z_{i}$ is a Zariski-closed subset of $|\mathfrak{X}|)$ then $\mathfrak{X}_{/ \mathcal{Z}}$ is the completion of $\mathfrak{X}$ along $Z$.

EXAMPLE 4.6.1. We give an example of $\mathcal{Z}=\mathscr{I}^{\phi}$ for $\mathfrak{X}=\widehat{\mathscr{S}}_{\mathrm{K}, W}$. For an isogeny class $\phi$, set $\mathscr{I}^{\phi}:=\left\{Z_{i}\right\}_{i \in \mathbf{I}}$, where $\mathbf{I}$ be the set of $I^{\phi}(\mathbb{Q})$-orbits of irreducible components of $\mathrm{RZ}_{G, b} \times G\left(\mathbb{A}_{\mathrm{f}}^{p}\right) / \mathrm{K}^{p}$, and $Z_{i} \subset\left|\widehat{\mathscr{S}}_{\mathrm{K}, W}\right|$ for $i \in \mathbf{I}$ is the image by $\Theta^{\phi}$ of the $I^{\phi}(\mathbb{Q})$-orbit of irreducible components corresponding to $i \in \mathbf{I}$. To see that $Z_{i}$ is a closed subset, note that any irreducible component of $\mathrm{RZ}_{G, b}^{\mathrm{red}}$ is projective. One can check that any $Z_{i}$ intersects with only finitely many $Z_{j}$ 's from (4.5.1). Therefore we can define $\left(\widehat{\mathscr{S}}_{\mathrm{K}, W}\right)_{/ \mathscr{I} \phi}$ as in Definition 4.6. 
The following theorem is a Hodge-type generalization of the unramified case of [26, Theorem 6.23].

Theorem 4.7. The morphism $\Theta^{\phi}$, obtained in Proposition 4.5, induces an isomorphism of formal schemes respecting the natural Weil descent datum over $\mathscr{O}_{E, \mathfrak{p}}$ :

$$
\Theta^{\phi}: I^{\phi}(\mathbb{Q}) \backslash \mathrm{RZ}_{G, b} \times G\left(\mathbb{A}_{\mathrm{f}}^{p}\right) / \mathrm{K}^{p} \stackrel{\sim}{\rightarrow}\left(\widehat{\mathscr{S}}_{\mathrm{K}, W}\right)_{/ \mathscr{I} \phi} .
$$

Proof. Note that $\Theta^{\phi}$ in the statement is a formally étale surjective monomorphism which induces a proper morphism on the underlying reduced schemes. Such a morphism between locally noetherian formal schemes is an isomorphism; see the proof of Theorem 6.23 in [26, p. 290].

For the remainder of the section, we prove some refinements of Theorem 4.7; namely, we descend the isomorphism $\Theta^{\phi}$ in Theorem 4.7 over $\mathscr{O}_{E, \mathfrak{p}}$ (not just over $W=W\left(\overline{\mathbb{F}}_{p}\right)$ ). For this, we need the following theorem of Kisin (which is highly nontrivial in the non-PEL case):

Theorem 4.8 (Kisin). The group $I^{\phi}(\mathbb{Q})$ as in Definition 4.4 is the $\mathbb{Q}$-points of reductive $\mathbb{Q}$-group $I^{\phi}$, which is an inner form of some Levi subgroup of $G$. More precisely, there exists an element $\gamma_{0} \in G(\mathbb{Q})$ such that $I^{\phi}$ is an inner form of the centralizer $G_{\gamma_{0}} \subset G$ of $\gamma_{0}$.

Furthermore, we have $I_{\mathbb{Q}_{p}}^{\phi} \subset J_{b}$, and $g \in J_{b}\left(\mathbb{Q}_{p}\right)$ lies in $I^{\phi}\left(\mathbb{Q}_{p}\right)$ if and only if $g$ corresponds to a quasi-isogeny of $\mathcal{A}_{\mathrm{K}, x}\left[p^{\infty}\right]$ defined over some finite field.

Proof. This is a direct consequence of [15, Corollaries 2.3.1 and 2.3.5].

Note that the closed immersion $I_{\mathbb{Q}_{p}}^{\phi} \subset J_{b}$ may not be an isomorphism in general. On the other hand, if $b$ is basic then one can show that $I_{\mathbb{Q}_{p}}^{\phi} \cong J_{b}$; cf. [32, Lemma 7.2.14].

4.9. Effectivity of Weil descent. Although the Weil descent datum $\Phi$ on $\mathrm{RZ}_{G, b}$ is not effective, we show that $\Phi$ induces an effective Weil descent datum on $I^{\phi}(\mathbb{Q}) \backslash \mathrm{R} Z_{G, b} \times G\left(\mathbb{A}_{\mathrm{f}}^{p}\right) / \mathrm{K}^{p}$. In particular, by Theorem $4.7\left(\widehat{\mathscr{S}}_{\mathrm{K}, W}\right)_{/ \mathscr{I} \phi}$ descends over $\operatorname{Spf} \mathscr{O}_{E, \mathrm{p}}$; cf. Corollary 4.9.3. In the PEL case, this result can be obtained from [26, Theorem 3.49 and Proposition 6.16].

By Kottwitz' theorem (Proposition 2.6.2), we may assume that $b \in G\left(K_{0}\right)$ satisfies the equation $(b \sigma)^{r}=\left(r v_{b}\right)(p) \sigma^{r}$ by replacing $b$ up to $\sigma$-conjugacy in $G\left(K_{0}\right)$. Viewing $\left(r v_{b}\right)(p) \in J_{b}\left(\mathbb{Q}_{p}\right)$ as a quasi-isogeny of $\mathbb{X}$ (cf. Proposition 2.6.5), the height of $\left(r v_{b}\right)(p)$ is precisely $r \operatorname{dim} \mathbb{X}$. (Note that we work with contravariant 
Dieudonné theory, while the formula in [26, Section 3.41] is deduced via covariant Dieudonné theory.) Therefore, we have an isomorphism

$$
\left\langle\left(r v_{b}\right)(p)\right\rangle \backslash \mathrm{R} Z_{G, b} \cong \coprod_{h=0}^{r \operatorname{dim} X_{0}-1} \mathrm{RZ} Z_{G, b}(h),
$$

where $\mathrm{RZ}_{G, b}(h)$ is a quasicompact open and closed formal subscheme defined by requiring the height of the quasi-isogeny to be $h \in \mathbb{Z}$.

Since $\left(r v_{b}\right)(p)$ is in the centre of $J_{b}\left(\mathbb{Q}_{p}\right)$ (cf. Proposition 2.6.5), the natural left action of $J_{b}\left(\mathbb{Q}_{p}\right)$ on $\mathrm{RZ}_{G, b}$ descends to the quotient, and the Weil descent datum $\Phi$ on $\mathrm{RZ}_{G, b}$ induces a Weil descent datum on this quotient.

Proposition 4.9.1. The Weil descent datum $\Phi$ on $\left\langle\left(r v_{b}\right)(p)\right\rangle \backslash \mathrm{RZ}_{G, b}$ is effective for any $r \in \mathbb{Z}$ such that $r v_{b}: \mathbf{D} \rightarrow G_{K_{0}}$ factors through $\mathbb{G}_{m}$ (via the natural projection $\left.\mathbf{D} \rightarrow \mathbb{G}_{m}\right)$.

Proof. Note that the closed immersion $\mathrm{RZ}_{G, b} \hookrightarrow \mathrm{RZ}_{b}$ commutes with the Weil descent datum $\Phi$ over $\mathscr{O}_{E, \mathfrak{p}}$, so it suffices to prove the claim for $\mathrm{RZ}_{b}$ instead of $R Z_{G, b}$. The case of $\mathrm{RZ}_{b}$ was already handled in [26, Theorem 3.49].

Next, we would like to approximate a suitable power of $\left(r v_{b}\right)(p)$ to a global element. By considering the image of $x \in \mathscr{S}_{\mathrm{K}}\left(\overline{\mathbb{F}}_{p}\right)$ in the Siegel modular variety $x \in \mathscr{S}_{\mathrm{K}^{\prime}}\left(\overline{\mathbb{F}}_{p}\right)$, we may apply [26, Lemma 6.17] to obtain that $\left(r v_{b}\right)(p) \in$ $\left(\operatorname{End}\left(\mathcal{A}_{\mathrm{K}, x}\right) \otimes \mathbb{Q}_{p}\right)^{\times}$. In particular, $\left(r v_{b}\right)(p) \in J_{b}\left(\mathbb{Q}_{p}\right)$ corresponds to a self quasi-isogeny of $\mathcal{A}_{\mathrm{K}, x}\left[p^{\infty}\right]$ defined over some finite field, so by Kisin's theorem (Theorem 4.8) we have $\left(r v_{b}\right)(p) \in I^{\phi}\left(\mathbb{Q}_{p}\right)$.

Now, let $Z^{\phi} \subset I^{\phi}$ denote the centre. Since $\left(r v_{b}\right)(p)$ is in the centre of $J_{b}\left(\mathbb{Q}_{p}\right)$, it follows that $\left(r v_{b}\right)(p) \in Z^{\phi}\left(\mathbb{Q}_{p}\right)$, which is contained in $Z^{\phi}\left(\mathbb{A}_{\mathrm{f}}\right)$.

Set $U^{\phi, p}=Z^{\phi}\left(\mathbb{A}_{\mathrm{f}}^{p}\right) \cap \mathrm{K}^{p}$ where the intersection is taken inside $G\left(\mathbb{A}_{\mathrm{f}}^{p}\right)$, and choose an open compact subgroup $U_{p}^{\phi} \subset Z^{\phi}\left(\mathbb{Q}_{p}\right)$ so that it is contained in the open compact subgroup of $J_{b}\left(\mathbb{Q}_{p}\right)$ consisting of automorphisms of $\mathbb{X}$. Since $U^{\phi}:=$ $U_{p}^{\phi} U^{\phi, p}$ is an open compact subgroup of $Z^{\phi}\left(\mathbb{A}_{\mathrm{f}}\right)$, the following abelian group

$$
Z^{\phi}(\mathbb{Q}) \backslash Z^{\phi}\left(\mathbb{A}_{\mathrm{f}}\right) / U^{\phi}
$$

is finite. We may assume that $\left(r v_{b}\right)(p) \in Z^{\phi}(\mathbb{Q}) \cdot U^{\phi}$ by replacing $r$ with a suitable integer multiple of $r$. Therefore, we may (and do) choose $r \in \mathbb{Z}$, so that there exists $z \in Z^{\phi}(\mathbb{Q})$ with $z \equiv\left(r v_{b}\right)(p) \bmod U^{\phi}$.

We have just proved the following proposition, which generalizes [26, Proposition 6.16]:

Proposition 4.9.2. The map $\Theta^{\phi}: \mathrm{RZ}_{G, b} \times G\left(\mathbb{A}_{\mathrm{f}}^{p}\right) / \mathrm{K}^{p} \rightarrow \widehat{\mathscr{S}}_{\mathrm{K}, W}$ (cf. Corollary 4.3.2) factors through $\left\langle\left(r v_{b}\right)(p)\right\rangle \backslash \mathrm{RZ}_{G, b} \times G\left(\mathbb{A}_{\mathrm{f}}^{p}\right) / \mathrm{K}^{p}$, where $r$ is chosen as above. 
The following Corollary is straightforward from Propositions 4.9.1 and 4.9.2:

Corollary 4.9.3. The Weil descent datum $\Phi$ on $I^{\phi}(\mathbb{Q}) \backslash R Z_{G, b} \times G\left(\mathbb{A}_{\mathrm{f}}^{p}\right) / K^{p}$ is effective.

\section{Rapoport-Zink uniformization via rigid geometry}

We continue to assume that $(G, \mathfrak{H})$ is a Hodge-type Shimura datum such that $G$ is unramified at $p$. Using our results in Section 4 for hyperspecial maximal level at $p$ we can obtain a rigid analytic uniformization result for other levels at $p$ (Theorem 5.4), generalizing the unramified case of [26, Theorem 6.36].

We continue to assume that $p>2$ without mentioning it.

\subsection{Level structures at $p$ for Hodge-type Shimura varieties in} characteristic 0. In Sections 3.2.3 and 3.2.4, we described level structures at $p$ and $G\left(\mathbb{Q}_{p}\right)$-action for Hodge-type Shimura varieties in characteristic 0 , working with abelian varieties up to isogeny. Here, we reformulate them only using prime-to- $p$ isogeny classes (so that we can relate it to the rigid analytic tower over $\mathrm{RZ}_{G, b}^{\text {rig }}$ ). We assume that $(G, \mathfrak{H})$ is of Hodge type with $G$ unramified at $p$, and make auxiliary choices as in Section 3.3.1.

Let $\mathrm{K}_{p}$ be an open compact subgroup of $G\left(\mathbb{Z}_{p}\right)$. For example, we may consider $\mathrm{K}_{p}^{(0)}:=G\left(\mathbb{Z}_{p}\right)$ and $\mathrm{K}_{p}^{(i)}:=\operatorname{ker}\left(G\left(\mathbb{Z}_{p}\right) \rightarrow G\left(\mathbb{Z} / p^{i}\right)\right)$ for $i>0$. Let $\mathrm{K}:=\mathrm{K}_{p} \mathrm{~K}^{p}$, and consider $\left(\mathcal{A}_{\mathrm{K}, E}, \eta_{\mathrm{K}}\right)$ where $\mathcal{A}_{\mathrm{K}, E}$ is viewed up to isogeny and $\eta_{\mathrm{K}}$ is as in Section 3.2.3. We can decompose $\eta_{\mathrm{K}}$ into the prime-to- $p$ part $\eta_{\mathrm{K}^{p}}$ (3.3.5) and the p-part

$$
\eta_{\mathrm{K}_{p}} \in \Gamma\left(\operatorname{Sh}_{\mathrm{K}_{p}^{(i)} \mathrm{K}^{p}}, \operatorname{isom}\left[\left(V_{\mathbb{Q}_{p}},\left(s_{\alpha}\right)\right),\left(\mathcal{V}_{\mathbb{Q}_{p}}\left(\mathcal{A}_{\mathrm{K}_{p}^{(i)} K^{p}, E}\right),\left(t_{\alpha, \text { ét }, p}^{\text {univ }}\right)\right)\right] / \mathrm{K}_{p}\right) .
$$

In the isogeny class of $\mathcal{A}_{\mathrm{K}, E}$, consider the pull-back of the abelian scheme $\mathcal{A}_{\mathrm{K}_{p}^{(0)} \mathrm{K}^{p}, E}$, up to prime-to- $p$ isogeny, that extends to the integral canonical model. We also denote it by $\mathcal{A}_{\mathrm{K}, E}$. Then $\eta_{\mathrm{K}_{p}}$ can be viewed as a right $\mathrm{K}_{p}$-coset of isomorphisms $\Lambda \stackrel{\sim}{\rightarrow} T_{p}\left(\mathcal{A}_{\mathrm{K}, E}\right)$ matching tensors. With such identification, we obtain the following description of $\mathrm{Sh}_{\mathrm{K}_{p} \mathrm{~K}^{p}}$ :

$$
\mathrm{Sh}_{\mathrm{K}_{p} \mathrm{~K}^{p}} \stackrel{\sim}{\rightarrow} \operatorname{isom}_{\mathrm{Sh}_{\mathrm{K}_{p}^{(0)} \mathrm{K}^{p}}}\left(\left[\Lambda_{\mathbb{Z}_{p}},\left(s_{\alpha}\right)\right],\left[T_{p}\left(\mathcal{A}_{\mathrm{K}_{p}^{(0)} \mathrm{K}^{p}, E}\right),\left(t_{\alpha, \text { ét }, p}^{\text {univ }}\right)\right]\right) / \mathrm{K}_{p},
$$

where the morphism is defined by restricting $\eta_{\mathrm{K}_{p}}$ to $\Lambda_{\mathbb{Z}_{p}}$.

When $\mathrm{K}_{p}=\mathrm{K}_{p}^{(i)}$ for some $i$, then (5.1.2) can be interpreted as follows:

$$
\operatorname{Sh}_{\mathrm{K}_{p}^{(i)} \mathrm{K}^{p}} \stackrel{\sim}{\rightarrow} \operatorname{isom}_{\mathrm{Sh}_{\mathrm{K}_{p}^{(0)} K^{p}}}\left(\left[\Lambda_{\mathbb{Z}_{(p)}} / p^{i},\left(s_{\alpha}\right)\right],\left[\mathcal{A}_{\mathrm{K}_{p}^{(0)} K^{p}, E}\left[p^{i}\right],\left(t_{\alpha, \mathrm{é}, p}^{\text {univ }}\right)\right]\right) .
$$


For $g \in G\left(\mathbb{Q}_{p}\right)$, assume that ${ }^{g} \mathrm{~K}_{p} \subset G\left(\mathbb{Z}_{p}\right)$. (This can be arranged by replacing $\mathrm{K}_{p}$ by a finite-index open subgroup; namely, $G\left(\mathbb{Z}_{p}\right) \cap{ }^{g} \mathrm{~K}_{p}$.) In Section 3.2.4, we showed that pulling back by $[g]: \mathrm{Sh}_{g_{\mathrm{K}} \mathrm{K}^{p}} \stackrel{\sim}{\rightarrow} \mathrm{Sh}_{\mathrm{K}_{p} \mathrm{~K}^{p}}$, we have $[g]^{*} \mathcal{A}_{\mathrm{K}_{p} \mathrm{~K}^{p}} \sim \mathcal{A}_{g_{\mathrm{K}_{p} \mathrm{~K}^{p}}}$ up to isogeny, and changes the level structure at $p$ by 'right translation by $g$ '. To translate this in terms of the level structure at $p$ described as in (5.1.2), the prime-to- $p$ isogeny class of $[g]^{*} \mathcal{A}_{\mathrm{K}_{p} \mathrm{~K}^{p}}$ is the unique one in the isogeny class of $\mathcal{A}_{s_{\mathrm{K}} \mathrm{K}^{p}}$ which matches the $\mathbb{Z}_{p}$-lattices $\Lambda_{\mathbb{Z}_{p}}$ and $T_{p}\left([g]^{*} \mathcal{A}_{\mathrm{K}_{p} \mathrm{~K}^{p}}\right)$ via $[g]^{*} \eta_{\mathrm{K}_{p}}$, and then $[g]^{*} \eta_{\mathrm{K}_{p}}$ defines a section of the right hand side of (5.1.2).

5.2. Rigid analytic tower of Hodge-type Rapoport-Zink spaces. Since $\mathrm{RZ}_{G, b}$ is locally formally of finite type over $\operatorname{Spf} W$, it is possible to associate the 'rigid analytic generic fibre', denoted by $\mathrm{RZ}_{G, b}^{\mathrm{rig}}$.

We use the notation from Section 5.1, such as $\mathrm{K}_{p}^{(i)} \subset G\left(\mathbb{Z}_{p}\right)$, and set $\mathrm{RZ}_{G, b}^{\mathrm{K}_{p}^{(0)}}:=$ $\mathrm{RZ}_{G, b}^{\mathrm{rig}}$. For any $\mathrm{K}_{p} \subset \mathrm{K}_{p}^{(0)}$, we now define, in a way analogous to (5.1.2), the following rigid analytic étale cover of $\mathrm{RZ}_{G, b}^{\mathrm{rig}}$ :

$$
\mathrm{RZ}_{G, b}^{\mathrm{K}_{p}}:=\operatorname{isom}_{\mathrm{Rz}}^{\mathrm{rig}}\left(\left[\Lambda_{\mathbb{Z}_{p}},\left(s_{\alpha}\right)\right],\left[T_{p}\left(X_{G, b}\right),\left(t_{\alpha, \text { ét }, p}^{\text {univ }}\right)\right]\right) / \mathrm{K}_{p},
$$

where $X_{G, b}$ is the universal $p$-divisible group over $\mathrm{RZ}_{G, b}, T_{p}\left(X_{G, b}\right)=$ $\left\{X_{G, b}\left[p^{n}\right]^{\text {rig }}\right\}$ is the $\mathbb{Z}_{p}$-local system over $\mathrm{RZ}_{G, b}^{\text {rig }}$ (that is, the Tate module of $\left.X_{G, b}\right)$, and $\left(t_{\alpha, \text { ét, } p}^{\text {univ }}: \mathbf{1} \rightarrow T_{p}\left(X_{G, b}\right)^{\otimes}\right)$ are the étale tensors associated to the 'universal crystalline tensors'. (See [12, Section 7.4] for more details. In [12] the tensors $\left(t_{\alpha, \text { ét, } p}^{\text {univ }}\right)$ were denoted as $\left(t_{\alpha, \text { ét }}\right)$.)

When $\mathrm{K}_{p}=\mathrm{K}_{p}^{(i)}$ for some $i$, then we have

$$
\mathrm{RZ}_{G, b}^{\mathrm{K}_{p}^{(i)}}:=\operatorname{isom}_{\mathrm{Rz}}^{\mathrm{rig}, b}\left(\left[\Lambda_{\mathbb{Z}_{(p)}} / p^{i},\left(s_{\alpha}\right)\right],\left[X_{G, b}\left[p^{i}\right],\left(t_{\alpha, \mathrm{ét}, p}^{\text {univ }}\right)\right]\right) .
$$

It is possible to extend the Galois action of $G\left(\mathbb{Z}_{p}\right)$ on the tower $\left\{\mathrm{RZ}_{G, b}^{\mathrm{K}_{p}}\right\}_{K_{p}}$ naturally to a $G\left(\mathbb{Q}_{p}\right)$-action in a way that is analogous to the case of Shimura varieties as discussed in Section 5.1; cf. [12, Section 7.4].

5.3. Rigid analytic Rapoport-Zink uniformization. We write $\mathrm{K}:=\mathrm{K}_{p} \mathrm{~K}^{p}$ with $\mathrm{K}_{p}=G\left(\mathbb{Z}_{p}\right)$. For an isogeny class $\phi$ of $\overline{\mathbb{F}}_{p}$-points of $\mathscr{S}_{\mathrm{K}}$, we set

$$
\operatorname{Sh}_{\mathrm{K}}^{\mathrm{rig}}(\phi):=\left(\left(\widehat{\mathscr{S}}_{\mathrm{K}, W}\right)_{/ \mathscr{I} \phi}\right)^{\mathrm{rig}} .
$$

If $\mathscr{I}^{\phi}$ is a finite collection of irreducible subvarieties of $\mathscr{S}_{\mathrm{K}, \overline{\mathbb{F}}_{p}}$, then $\operatorname{Sh}_{\mathrm{K}}^{\text {rig }}(\phi)$ is the tube of $\mathscr{I}^{\phi}$ in $\widehat{\mathscr{S}}_{\mathrm{K}, W}$. In general, $\mathrm{Sh}_{\mathrm{K}}^{\text {rig }}(\phi)$ is a union of tubes of the irreducible subvarieties $Z \in \mathscr{I}^{\phi}$. 
Since the construction of rigid analytic generic fibre is functorial, we obtain the following maps of rigid analytic spaces over $K_{0}$ from Theorem 4.7 for $\mathrm{K}:=$ $\mathrm{K}_{p}^{(0)} \mathrm{K}^{p}$ :

$$
\Theta^{\phi, \text { rig }}: I^{\phi}(\mathbb{Q}) \backslash \mathrm{RZ} Z_{G, b}^{\text {rig }} \times G\left(\mathbb{A}_{\mathrm{f}}^{p}\right) / \mathrm{K}^{p} \stackrel{\sim}{\rightarrow} \operatorname{Sh}_{\mathrm{K}}^{\text {rig }}(\phi) .
$$

Furthermore, the rigid analytic spaces and the maps in (5.3.2) descend over $E_{\mathfrak{p}}$ by Corollary 4.9.3.

From now on, assume that $\mathrm{K}:=\mathrm{K}_{p} \mathrm{~K}^{p} \subset G\left(\mathbb{A}_{\mathrm{f}}\right)$ such that $\mathrm{K}_{p} \subset \mathrm{K}_{p}^{(0)}=G\left(\mathbb{Z}_{p}\right)$, and $\mathrm{K} \subset G\left(\mathbb{A}_{\mathrm{f}}\right)$ is a 'small enough'. We let $\mathrm{Sh}_{\mathrm{K}, K_{0}}^{\text {rig }}$ and $S h_{\mathrm{K}, E_{\mathrm{p}}}^{\text {rig }}$ respectively denote the rigid analytifications of $S h_{K}, K_{0}$ and $S h_{K}, E_{\mathfrak{p}}$.

DEFINITION 5.3.3. We let $\operatorname{Sh}_{\mathrm{K}}^{\text {rig }}(\phi)$ denote the preimage of $\operatorname{Sh}_{\mathrm{K}_{p}^{(0)} K^{p}}^{\text {rig }}(\phi)$ via the natural projection map $\mathrm{Sh}_{\mathrm{K}_{p} K^{p}, K_{0}}^{\mathrm{rig}} \rightarrow \mathrm{Sh}_{\mathrm{K}_{p}^{(0)} K^{p}, K_{0}}^{\text {rig }}$. Equivalently, by (5.1.2) we have

$$
\operatorname{Sh}_{\mathrm{K}_{p} K^{p}}^{\mathrm{rig}}(\phi) \cong \operatorname{isom}_{\mathrm{Sh}_{\mathrm{K}_{p}^{(0)} K^{p}}^{\mathrm{rig}}(\phi)}\left(\left[\Lambda_{\mathbb{Z}_{p}},\left(s_{\alpha}\right)\right],\left[T_{p}\left(\mathcal{A}_{\mathrm{K}_{p}^{(0)} \mathrm{K}^{p}}\right),\left(t_{\alpha, \text { ét, } p}^{\mathrm{univ}}\right)\right]\right) / \mathrm{K}_{p} .
$$

Since $\operatorname{Sh}_{K_{p}^{(0)} K^{p}}^{\text {rig }}(\phi)$ is defined over $E_{\mathfrak{p}}$ (by Corollary 4.9.3), it follows that $\mathrm{Sh}_{\mathrm{K}_{p} \mathrm{~K}^{p}}^{\mathrm{rig}}(\phi)$ is also defined over $E_{\mathfrak{p}}$.

By matching the definitions of the coverings $\operatorname{Sh}_{K_{p} K^{p}}^{\text {rig }}(\phi) \rightarrow S h_{K_{p}^{(0)} K^{p}}^{\text {rig }}(\phi)$ (Definition 5.3.3) and $\mathrm{RZ}_{G, b}^{\mathrm{K}_{p}} \rightarrow \mathrm{RZ} \mathrm{r}_{G, b}^{\text {rig }}$ (5.2.1), we obtain the following theorem:

Theorem 5.4. Assume that $\mathrm{K}:=\mathrm{K}_{p} \mathrm{~K}^{p} \subset G\left(\mathbb{A}_{\mathrm{f}}\right)$ such that $\mathrm{K}_{p} \subset \mathrm{K}_{p}^{(0)}=G\left(\mathbb{Z}_{p}\right)$. Then, we can lift $\Theta^{\phi, \text { rig }}$ (5.3.2) to

$$
\Theta_{\mathrm{K}}^{\phi}: I^{\phi}(\mathbb{Q}) \backslash \mathrm{R} Z_{G, b}^{\mathrm{K}_{p}} \times G\left(\mathbb{A}_{\mathrm{f}}^{p}\right) / \mathrm{K}^{p} \stackrel{\sim}{\rightarrow} \operatorname{Sh}_{\mathrm{K}}^{\text {rig }}(\phi),
$$

which also descends over $E_{\mathfrak{p}}$. Furthermore, by varying $\mathrm{K}_{p}$ and $\mathrm{K}^{p}$, the isomorphism $\left\{\Theta_{\mathbf{K}}^{\phi}\right\}$ is equivariant for the $G\left(\mathbb{A}_{\mathrm{f}}\right)$-action. (On the left hand side, $G\left(\mathbb{Q}_{p}\right)$ acts naturally on $\left\{\mathrm{RZ}_{G, b}^{\mathrm{K}_{p}}\right\}_{\mathrm{K}_{p}}$, and $G\left(\mathbb{A}_{\mathrm{f}}^{p}\right)$ acts by left translation on $\left\{G\left(\mathbb{A}_{\mathrm{f}}^{p}\right) / \mathrm{K}^{p}\right\}_{K^{p}}$. On $\left\{\operatorname{Sh}_{\mathrm{K}}^{\mathrm{rig}}(\phi)\right\}_{\mathrm{K}}$, the $G\left(\mathbb{A}_{\mathrm{f}}\right)$-action is the restriction on the natural $G\left(\mathbb{A}_{\mathrm{f}}\right)$-action on $\left\{\mathrm{Sh}_{\mathrm{K}}\right\}_{\mathrm{K}}$.)

\section{Acknowledgements}

The author would like to thank George Pappas for his careful reading and providing comments. He also would like to thank Xinwen Zhu for informing him of [32]. While the paper was being written and revised, the author was supported by Herchel Smith Postdoctoral Fellowship and the EPSRC (Engineering and Physical Sciences Research Council) in the form of EP/L025302/1. 


\section{References}

[1] P. Berthelot, L. Breen and W. Messing, Théorie de Dieudonné Cristalline. II, Lecture Notes in Mathematics, 930 (Springer, Berlin, 1982).

[2] D. Blasius, 'A $p$-adic property of Hodge classes on abelian varieties, Motives (Seattle, WA, 1991)', Proc. Symp. Pure Mathematics, 55 (American Mathematical Society, Providence, RI, 1994), 293-308.

[3] A. Borel, Linear Algebraic Groups, 2nd edn, Graduate Texts in Mathematics, 126 (Springer, New York, 1991).

[4] H. Carayol, 'Sur les représentations $l$-adiques associées aux formes modulaires de Hilbert', Ann. Sci. Éc. Norm. Supér. (4) 19(3) (1986), 409-468.

[5] M. Chen, M. Kisin and E. Viehmann, 'Connected components of affine Deligne-Lusztig varieties in mixed characteristic', Compos. Math. 151(9) (2015), 1697-1762.

[6] P. Deligne, Travaux de Shimura, Séminaire Bourbaki, 23ème année (1970/71, Exp. No. 389, Lecture Notes in Mathematics, 244 (Springer, Berlin, 1971), 123-165.

[7] P. Deligne, 'Hodge cycles on abelian varieties', in Hodge Cycles, Motives, and Shimura Varieties, Lecture Notes in Mathematics, 900 (Springer, Berlin, 1982), 9-100. Notes by J. S. Milne.

[8] G. Faltings, 'Integral crystalline cohomology over very ramified valuation rings', J. Amer. Math. Soc. 12(1) (1999), 117-144.

[9] B. Howard and G. Pappas, 'Rapoport-Zink spaces for spinor groups', Compos. Math. 153(5) (2017), 1050-1118.

[10] N. Katz, 'Serre-Tate local moduli', in Algebraic surfaces (Orsay, 1976-78), Lecture Notes in Mathematics, 868 (Springer, Berlin, 1981), 138-202.

[11] W. Kim, 'The relative Breuil-Kisin classification of $p$-divisible groups and finite flat group schemes', Int. Math. Res. Not. IMRN (17) (2015), 8152-8232.

[12] W. Kim, 'Rapoport-Zink spaces of Hodge type', Forum Math. Sigma 6 (2018), e8, 110.

[13] W. Kim and K. Madapusi Pera, '2-adic integral canonical models', Forum Math. Sigma 4(e28) (2016), 34.

[14] M. Kisin, 'Integral models for Shimura varieties of abelian type', J. Amer. Math. Soc. 23(4) (2010), 967-1012.

[15] M. Kisin, 'mod $p$ points on Shimura varieties of abelian type', J. Amer. Math. Soc. 30(3) (2017), 819-914.

[16] M. Kisin and G. Pappas, 'Integral models of Shimura varieties with parahoric level structure', Publ. Math. Inst. Hautes Études Sci. (First Online) (2018), 1-98, doi:10.1007/s10240-018-0100-0.

[17] R. E. Kottwitz, 'Shimura varieties and twisted orbital integrals', Math. Ann. 269(3) (1984), 287-300.

[18] R. E. Kottwitz, 'Isocrystals with additional structure', Compos. Math. 56(2) (1985), 201-220.

[19] R. E. Kottwitz, 'Points on some Shimura varieties over finite fields', J. Amer. Math. Soc. 5(2) (1992), 373-444.

[20] G. Laumon and L. Moret-Bailly, 'Champs algébriques', in Ergebnisse der Mathematik und ihrer Grenzgebiete. 3. Folge. A Series of Modern Surveys in Mathematics, [Results in Mathematics and Related Areas. 3rd Series, A Series of Modern Surveys in Mathematics], 39 (Springer, Berlin, 2000).

[21] B. Mazur and W. Messing, Universal Extensions and One Dimensional Crystalline Cohomology, Lecture Notes in Mathematics, 370 (Springer, Berlin, 1974). 
[22] W. Messing, The crystals associated to Barsotti-Tate groups: with applications to abelian schemes, Lecture Notes in Mathematics, 264 (Springer, Berlin, 1972).

[23] J. S. Milne, Shimura varieties and motives, Motives (Seattle, WA, 1991), Proc. Symp. Pure Math., 55 (American Mathematical Society, Providence, RI, 1994), 447-523.

[24] J. S. Milne, 'Introduction to Shimura varieties', in Harmonic Analysis, the Trace Formula, and Shimura Varieties, Clay Mathematics Proceedings, 4 (American Mathematical Society, Providence, RI, 2005), 265-378.

[25] B. Moonen, 'Models of Shimura varieties in mixed characteristics', in Galois Representations in Arithmetic Algebraic Geometry (Durham, 1996), London Mathematical Society, Lecture Note Series, 254 (Cambridge University Press, Cambridge, 1998), 267-350.

[26] M. Rapoport and T. Zink, Period Spaces for p-Divisible Groups, Annals of Mathematics Studies, 141 (Princeton University Press, Princeton, NJ, 1996).

[27] A. Vasiu, 'Integral canonical models of Shimura varieties of preabelian type', Asian J. Math. 3(2) (1999), 401-518.

[28] A. Vasiu, Good reductions of Shimura varieties of Hodge type in arbitrary unramified mixed characteristic, part I, Preprint, 2007, arXiv:0707.1668, 53 pages.

[29] A. Vasiu, 'Good reductions of Shimura varieties of Hodge type in arbitrary unramified mixed characteristic', Part II, Preprint, 2007, arXiv:0712.1572, 29 pages.

[30] A. Vasiu, 'A motivic conjecture of Milne', J. Reine Angew. Math. 685 (2013), 181-247.

[31] T. Wedhorn, 'On Tannakian duality over valuation rings', J. Algebra 282(2) (2004), 575-609.

[32] L. Xiao and X. Zhu, 'Cycles on Shimura varieties via geometric Satake', Preprint, 2017, arXiv:1707.05700. 